\title{
A Petrov-Galerkin multilayer discretization to second order elliptic boundary value problems
}

\author{
Toms Chacón Rebollo ${ }^{\mathrm{a}}$, Daniel Franco Coronil ${ }^{\mathrm{b}, *}$, Fréderic Hecht ${ }^{\mathrm{c}}$ \\ ${ }^{a}$ Departamento EDAN \& IMUS, Universidad de Sevilla, C/Tarfia, s/n, 41012 Sevilla \\ (Spain) \\ ${ }^{b}$ Departamento EDAN, Universidad de Sevilla, C/Tarfia, s/n, 41012 Sevilla (Spain) \\ ${ }^{c}$ Laboratoire Jacques-Louis Lions, Université de Paris VI. 5 Place Jussieu, 75005 Paris \\ (France)
}

\begin{abstract}
We study in this paper a multilayer discretization of second order elliptic problems, aimed at providing reliable multilayer discretizations of shallow fluid flow problems with diffusive effects. This discretization is based upon the formulation by transposition of the equations. It is a Petrov-Galerkin discretization in which the trial functions are piecewise constant per horizontal layers, while the trial functions are continuous piecewise linear, on a vertically shifted grid.

We prove the well posedness and optimal error order estimates for this discretization in natural norms, based upon specific inf-sup conditions.

We present some numerical tests with parallel computing of the solution based upon the multilayer structure of the discretization, for academic problems with smooth solutions, with results in full agreement with the theory developed. Keywords: Multilayer methods, transposition solution, finite differences, Petrov-Galerkin discretizations 2010 MSC: $65 \mathrm{Nxx}, 65 \mathrm{Yxx}, 76 \mathrm{Mxx}$
\end{abstract}

\footnotetext{
*Departamento EDAN, Universidad de Sevilla, C/Tarfia, s/n, 41012 Sevilla (Spain)

Email addresses: chacon@us.es (Toms Chacón Rebollo), franco@us.es (Daniel Franco Coronil), hechteann.jussieu.fr (Fréderic Hecht)
} 


\section{Introduction and motivation}

This paper deals with the numerical approximation of the Poisson and related problems by means of layer-wise discontinuous solutions. It is motivated by the construction of multilayer discretizations of fluid flow equations on shallow domains. This produces a reduction of the dimensionality of the approximated problem, yielding in practice a domain decomposition discretization that is solved by parallel procedures.

This is the case for instance of the works of Fernndez-Nieto and co-workers [1, 2] and of Sainte-Marie and co-workers [3] to solve the Navier-Stokes equations. In these papers the velocity is discretized by layer-wise smooth functions, discontinuous across the layer boundaries. The conservation of momentum needs the continuity of diffusive fluxes across layer boundaries. This is ensured in these papers by specific techniques based upon finite-difference discretizations in the vertical direction. Both papers perform stability analysis for the discretizations introduced, however no convergence proofs are reported. This mainly occurs because the discretizations are purely finite-difference approximations, that are not linked to variational formulation of the targeted equations.

In this paper we look for stable multilayer discretizations for which convergence proofs are reachable. More concretely, we look for stable discretizations that are related to variational formulation of the equations. A possible discretization meeting these criteria could be provided by the Discontinuous Galerkin (DG) method. This method was introduced in the 90's by Cockburn and Shu for hyperbolic conservation laws (cf. 4]), and later adapted to elliptic problems, by re-writing as two coupled first-order conservation laws for the unknown and its gradient (cf. [5, 6] and references therein). DG methods for elliptic problems are based upon the flux formulation of the elliptic problem, that includes both the original unknown so as its gradient as unknowns of the discretization. This allows to approximate both the unknown and its gradient by discontinuous finite elements. The stability of the formulation strongly depends on the choice of the numerical fluxes of both unknowns. 
An alternative discretization could be provided by the Discontinuous Petrov Galerkin methodology, introduced in a series of papers by Sacco and co-workers (cf. e.g. [7, 8) and systematically studied by Demkowicz and Gopalakrishnan (see [9, 10, 11, 12] and references therein). The DPG methodology approximates the unknown by either continuous or discontinuous trial functions, while the tests functions necessarily need to be discontinuous. This formulation admits three equivalent interpretations: a Petrov-Galerkin method with test functions that achieve the supremum in the inf-sup condition, a minimum-residual method with residual measured in a suitable dual norm, and a mixed formulation where one solves simultaneously for the Riesz representation of the residual. The stability of the formulation is a direct consequence of the first of these three interpretations.

Here we propose a discretization specifically adapted to multilayer discretizations, for elliptic problems related to the Poisson equations. The main idea is to start from the solution by transposition of the equations, much as the ultraweak formulation considered in the works 7,8 , by Sacco and co-workers. The solution by transposition naturally belongs to $L^{2}$ spaces, and thus admits piecewise discontinuous approximations. Based upon this procedure, we propose a Petrov-Galerkin discretization for cylindrical domains, in which the solution is a layer-wise constant function, while the test functions are continuous piecewise affine polynomials in the vertical direction, with knots in a shifted grid. We derive a single "recepy" to build this kind of discretizations: Approximate the vertical derivative of the unknown by the vertical derivative of its interpolate on the test functions space.

We prove the well-posedness of this discretization, given by an inf-sup condition satisfied by the bilinear form appearing in the discrete problem. Also, we prove optimal order error estimates for smooth solutions. We further extend the multilayer discretization technique introduced to domains which are vertical deformations of cylindrical domains, which appear in shallow water problems when a flat surface is deformed. We also prove well-posedness and optimal order error estimates for this extension. We further extend the method to Neumann 
boundary conditions, by slightly changing the test space.

We finally present some 3D numerical tests by parallel solution of the resulting linear system, taking advantage of the multilayer structure of the discretization, what allows to only solve $2 \mathrm{D}$ linear systems. These tests present speeds-ups rates ranging from 20 to 50 , while presenting optimal convergence orders for smooth functions, in agreement with the theoretical expectations.

The paper is organized as follows. Section 2 introduces the motivation and the basic multilayer discretization that we consider, for the Poisson problem in a cylindric domain. This discretization is studied in Section 3 where stability, convergence and obtention of error estimates are analyzed. In Section 4 the discretization is extended to Poisson problems in domains with non-flat upper boundary, also analyzing stability, convergence and error estimates. Section 5 is devoted to the extension of the discretization to Neumann boundary conditions. Finally Section 6 present several 3D numerical tests for each of the cases considered, for smooth solutions.

\section{Multilayer approximation}

Let us consider a cylindrical domain $\Omega=\omega \times(0, L)$ where $\omega \subset \mathbb{R}^{d}$ is bounded domain, and $d \geq 1$ is an integer number. Let us consider the homogeneous Dirichlet Poisson problem as a model problem:

$$
\left\{\begin{aligned}
-\Delta v & =f \text { in } \quad \Omega, \\
v & =0 \text { on } \partial \Omega .
\end{aligned}\right.
$$

As is standard, this problem can be written in variational form: Given $f \in$ $H^{-1}(\Omega)$, find $v \in H_{0}^{1}(\Omega)$ such that

$$
a(v, \varphi)=L(\varphi), \quad \forall \varphi \in H_{0}^{1}(\Omega),
$$

with

$$
a(v, \varphi)=\int_{\Omega} \nabla v \nabla \varphi d \Omega, \text { and } L(\varphi)=<f, \varphi>_{\Omega} .
$$


The solution of this problem is also the solution of the transposition formulation of problem (1), given by (Cf. [13]): Find $v \in L^{2}(\Omega)$ such that for all $\phi \in L^{2}(\Omega)$,

$$
\int_{\Omega} v \phi d \Omega=L(\mathcal{T} \phi)
$$

where $\mathcal{T} \phi=\varphi$ is the solution of

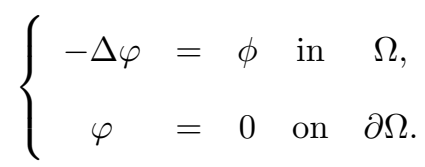

Problem (4) admits a unique solution in $L^{2}(\Omega)$, that necessarily coincides with the solution of (2). Also, problem (4) admits a unique solution when $f$ is less smooth (actually, when $\left.f \in\left(H_{0}^{1}(\Omega) \cap H^{2}(\Omega)\right)^{\prime}\right)$ whenever the problem (5) is regular, in the sense that $\varphi \in H_{0}^{1}(\Omega) \cap H^{2}(\Omega)$ (Cf. [13]).

However, we are not interested in approximating the formulation by transposition (4), rather we use it as a base to build our multilayer discretization of the weak standard formulation 2 . To do it, let us split the domain $\Omega$ along the vertical direction into $N \geq 1$ plane layers of constant thickness $h=L / N$ with $N+1$ interfaces $\Gamma_{\alpha+\frac{1}{2}}$ of equations $z=z_{\alpha+\frac{1}{2}}$ for $\alpha=0,1, \ldots, N$ (see Figure 1 . Hence, we split $\bar{\Omega}=\bigcup_{\alpha=1}^{N} \overline{\Omega_{\alpha}}$ with

$$
\Omega_{\alpha}=\left\{(x, z) ; x \in w \text { and } z_{\alpha-\frac{1}{2}}<z<z_{\alpha+\frac{1}{2}}\right\} \text {. }
$$

We assume $\partial \Omega=\Gamma_{b} \cup \Gamma_{s} \cup \Gamma_{l}$, where $\Gamma_{b}$ and $\Gamma_{s}$ are the boundaries of the bottom and the top, respectively, and $\Gamma_{l}$ is the vertical boundary of the domain. We shall denote by $z_{b}=z_{\frac{1}{2}}=0$ and $z_{s}=z_{N+\frac{1}{2}}=L$ the equations of the bottom and the top interfaces $\Gamma_{b}$ and $\Gamma_{s}$, respectively. Notice that $\partial \Omega_{\alpha}=$ $\Gamma_{\alpha-\frac{1}{2}} \cup \Gamma_{\alpha+\frac{1}{2}} \cup \Gamma_{l, \alpha}$, with

$$
\Gamma_{l, \alpha}=\left\{(x, z) \in \mathbb{R}^{d} ; x \in \partial w \text { and } z_{\alpha-\frac{1}{2}} \leq z \leq z_{\alpha+\frac{1}{2}}\right\} .
$$

Then the vertical boundary is split as $\Gamma_{l}=\bigcup_{\alpha=1}^{N} \Gamma_{l, \alpha}$. 


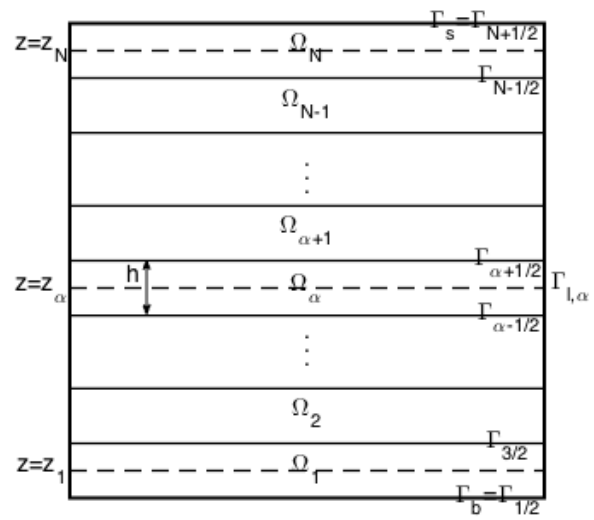

Figure 1: Splitting of the domain $\Omega$

Also, we denote $z_{\alpha}=\frac{z_{\alpha-\frac{1}{2}}+z_{\alpha+\frac{1}{2}}}{2}, \forall \alpha=1,2, \ldots, N$.

We shall assume from now on that $\omega$ is polygonal. Let us now introduce discretization space for the unknown $v$,

$$
X_{h}=\left\{v_{h} \in L^{2}(\Omega) / v_{h}=\sum_{\alpha=1}^{N} v_{h}^{\alpha} \otimes \kappa_{\alpha} \text { with } v_{h}^{\alpha} \in V_{0, k}(\omega)\right\},
$$

where $\kappa_{\alpha}$ is the characteristic function of the interval $\left(z_{\alpha-1 / 2}, z_{\alpha+1 / 2}\right)$ and $V_{0, k}(\omega)$ is a finite element sub-space of $H_{0}^{1}(\omega)$. The sub-index $k$ denotes the horizontal grid size. We assume that $V_{0, k}(\omega)=V_{k}(\omega) \cap H_{0}^{1}(\omega)$ where $V_{k}(\omega)$ is a Lagrange finite element space, constructed when $d=2$ with either triangular or quadrilateral elements, i. e., there exists a grid $\mathcal{M}_{k}$ such that

$$
V_{k}(\omega)=\left\{w_{h} \in C^{0}(\bar{\omega})\left|w_{h}\right|_{K} \in R_{l}(K), \forall K \in \mathcal{M}_{k}\right\}
$$

where $R_{l}(K)=\left\{\begin{array}{l}P_{l}(K) \text { if } \mathcal{M}_{k} \text { is formed by triangles } \\ Q_{l}(K) \text { if } \mathcal{M}_{k} \text { is formed by quadrilaterals, }\end{array}\right.$ for some integer $l \geq 1$; where $P_{l}(K)$ is the space of polynomials on $K$ of global degree less than or equal to $l$, and $Q_{l}(K)$ is the space of polynomials on $K$ of degree less than or 
equal to $l$ in each variable. When $d=1$, the triangulation is formed by segments $K$, and $R_{l}(K)=P_{l}(K)$.

We approximate the solution $v$ of problem (4) by some $v_{h} \in X_{h}$. Observe that for all $\varphi \in H^{2}(\Omega) \cap H_{0}^{1}(\Omega)$,

$$
\begin{aligned}
& -\int_{\Omega} v_{h} \Delta \varphi d \Omega=-\sum_{\alpha=1}^{N} \int_{\Omega_{\alpha}} v_{h}^{\alpha} \Delta \varphi d \Omega=-\sum_{\alpha=1}^{N} \int_{\partial \Omega_{\alpha}} v_{h}^{\alpha} \partial_{n} \varphi d \Gamma \\
& +\sum_{\alpha=1}^{N} \int_{\Omega_{\alpha}} \nabla v_{h}^{\alpha} \nabla \varphi d \Omega .
\end{aligned}
$$

As $\left.v_{h}^{\alpha}\right|_{\partial \omega}=0$, then it holds

$$
\int_{\partial \Omega_{\alpha}} v_{h}^{\alpha} \partial_{n} \varphi=\int_{\Gamma_{\alpha+\frac{1}{2}}} v_{h}^{\alpha} \partial_{z} \varphi-\int_{\Gamma_{\alpha-\frac{1}{2}}} v_{h}^{\alpha} \partial_{z} \varphi
$$

where $\partial_{z}$ denotes the vertical derivative. Thus,

$$
-\int_{\Omega} v_{h} \Delta \varphi d \Omega=a_{h}\left(v_{h}, \varphi\right)
$$

where $a_{h}$ is a the bilinear form defined by

$$
\begin{aligned}
a_{h}\left(v_{h}, \varphi\right)= & \sum_{\alpha=1}^{N}\left(\nabla v_{h}^{\alpha}, \nabla \varphi\right)_{\Omega_{\alpha}}+\sum_{\alpha=1}^{N-1} \int_{\Gamma_{\alpha+\frac{1}{2}}}\left(v_{h}^{\alpha+1}-v_{h}^{\alpha}\right) \partial_{z} \varphi d \Gamma \\
& -\int_{\Gamma_{s}} v_{h}^{N} \partial_{z} \varphi d \Gamma+\int_{\Gamma_{b}} v_{h}^{1} \partial_{z} \varphi d \Gamma ;
\end{aligned}
$$

Next, we introduce the discrete test functions space,

$$
Y_{h}=\left\{\varphi_{h} \in L^{2}(\Omega) / \varphi_{h}=\sum_{\alpha=1}^{N} \varphi^{\alpha} \otimes \sigma_{\alpha}, \text { with } \varphi^{\alpha} \in V_{0, k}(\omega)\right\},
$$

where $\sigma_{1}, \sigma_{2}, \cdots, \sigma_{N}$ are piecewise affine $1 \mathrm{D}$ functions on the intervals $\left[z_{b}, z_{1}\right]$, $\left[z_{1}, z_{2}\right], \cdots,\left[z_{N-1}, z_{N}\right],\left[z_{N}, z_{s}\right]$ that satisfy $\sigma_{i}\left(z_{j}\right)=\delta_{i j}$ and $\sigma_{i}\left(z_{b}\right)=\sigma_{i}\left(z_{s}\right)=0$ , for all $i, j=1,2, \ldots, N$, as we see in Figure 2, Then $Y_{h}$ is a subspace of $H_{0}^{1}(\Omega)$ formed by piecewise $\mathbb{P}_{1}$ polynomials in the variable $z$ with coefficients in $H_{0}^{1}(\omega)$. Note that the intervals $\left[z_{b}, z_{1}\right]$, and $\left[z_{N}, z_{s}\right]$ have length $h / 2$. Introducing them allows to have $\operatorname{dim}\left(Y_{h}\right)=\operatorname{dim}\left(X_{h}\right)$.

We now are in a position to state the multilayer discretization of (2) that we study in this paper,

$$
\left\{\begin{array}{l}
\text { Find } \quad v_{h} \in X_{h}, \quad \text { such that } \\
a_{h}\left(v_{h}, \varphi_{h}\right)=L\left(\varphi_{h}\right), \quad \forall \varphi_{h} \in Y_{h} .
\end{array}\right.
$$




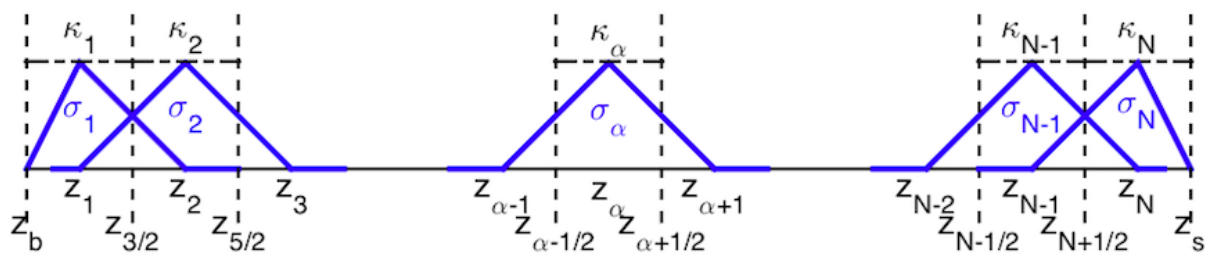

Figure 2: Basis function on $X_{h}$ and $Y_{h}$

Notice that although the space $Y_{h}$ is not a sub-set of $H_{0}^{1}(\Omega) \cap H^{2}(\Omega)$, the action $a_{h}\left(v_{h}, \varphi_{h}\right)$ is well defined whenever $v_{h} \in X_{h}$ and $\varphi_{h} \in Y_{h}$.

Observation 1. If we consider the mapping $\mathcal{T}_{h}: X_{h} \longrightarrow Y_{h}$ defined by

$$
\mathcal{T}_{h} v_{h}=\sum_{\alpha=1}^{N} v_{h}^{\alpha} \otimes \sigma_{\alpha} \quad \forall v_{h} \in X_{h},
$$

the form $a_{h}$ can be written on $Y_{h}$ as

$$
a_{h}\left(v_{h}, \varphi_{h}\right)=\int_{\Omega} \nabla_{H} v_{h} \nabla_{H} \varphi_{h}+\int_{\Omega} \partial_{z}\left(\mathcal{T}_{h}\left(v_{h}\right)\right) \partial_{z} \varphi_{h}
$$

where we denote $\nabla=\left(\nabla_{H}, \partial_{z}\right)$ with $\nabla_{H}=\left(\partial_{1}, \cdots, \partial_{d-1}\right)$ the horizontal gradient.

\section{Stability and convergence analysis}

In this section we study the well-posedness of problem (8) in the sense of Hadamard: We prove that it admits a unique solution that depends continuously on the data. This will be based upon the Banach-Neĉas-Babuska theorem (Cf. [14]).

We consider the space $X_{h}$ endowed with the following $H_{0}^{1}(\Omega)$ discrete norm:

$$
\begin{aligned}
& \left\|v_{h}\right\|_{X_{h}}=\left(h \sum_{\alpha=1}^{N}\left\|\nabla v_{h}^{\alpha}\right\|_{0, \omega}^{2}+\frac{h}{2}\left\|\frac{v_{h}^{1}}{h / 2}\right\|_{0, \omega}^{2}+\sum_{\alpha=1}^{N-1} h\left\|\frac{v_{h}^{\alpha+1}-v_{h}^{\alpha}}{h}\right\|_{0, \omega}^{2}\right. \\
& \left.+\frac{h}{2}\left\|\frac{v_{h}^{N}}{h / 2}\right\|_{0, \omega}^{2}\right)^{1 / 2}=\left(\left\|\nabla_{H} v_{h}\right\|_{0, \Omega}^{2}+\left\|\partial_{z}\left(\mathcal{T}_{h} v_{h}\right)\right\|_{0, \Omega}^{2}\right)^{1 / 2},
\end{aligned}
$$

and the space $Y_{h}$ endowed with the $H_{0}^{1}(\Omega)$ norm. It holds the 
Lemma 1. The mapping $\mathcal{T}_{h}$ is an isomorphism between normed spaces. Moreover, it holds

$$
\left\|\nabla\left(\mathcal{T}_{h} v_{h}\right)\right\|_{0, \Omega} \leq\left\|v_{h}\right\|_{X_{h}} \leq \sqrt{3}\left\|\nabla\left(\mathcal{T}_{h} v_{h}\right)\right\|_{0, \Omega}, \quad \forall v_{h} \in X_{h}
$$

Proof: By construction $\mathcal{T}_{h}$ is linear and bijective. To prove the equivalence of the norms, let $\varphi_{h} \in Y_{h}$. Denote $\varphi^{\alpha}=\varphi_{h}\left(z_{\alpha}\right)$. Some standard calculations yield

$$
\begin{aligned}
\left\|\nabla_{H} \varphi_{h}\right\|_{0, \Omega}^{2}= & \frac{h}{6} \int_{\omega}\left|\nabla_{H} \varphi^{1}\right|^{2}+\sum_{\alpha=1}^{N-1} \frac{h}{3}\left\{\int_{\omega}\left|\nabla_{H} \varphi^{\alpha}\right|^{2}+\int_{\omega}\left|\nabla_{H} \varphi^{\alpha+1}\right|^{2}\right\}+ \\
& +\sum_{\alpha=1}^{N-1} \frac{h}{3} \int_{\omega} \nabla_{H} \varphi^{\alpha} \cdot \nabla_{H} \varphi^{\alpha+1}+\frac{h}{6} \int_{\omega}\left|\nabla_{H} \varphi^{N}\right|^{2} .
\end{aligned}
$$

Also, as

$$
\partial_{z} \varphi_{h}=\left\{\begin{array}{cll}
\frac{\varphi^{1}}{h / 2} & \text { in } \omega \times\left[z_{b}, z_{1}\right], \\
\frac{\left(\varphi^{\alpha+1}-\varphi^{\alpha}\right)}{h} & \text { in } \omega \times\left[z_{\alpha}, z_{\alpha+1}\right], \quad \forall \alpha=1,2, \ldots, N-1 \\
\frac{-\varphi^{N}}{h / 2} & \text { in } \omega \times\left[z_{N}, z_{s}\right] ;
\end{array}\right.
$$

it follows

$$
\begin{gathered}
\left\|\nabla \varphi_{h}\right\|_{0, \Omega}^{2}=\frac{h}{2}\left\|\nabla_{H} \varphi^{1}\right\|_{0, \omega}^{2}+\sum_{\alpha=2}^{N-1} \frac{2 h}{3}\left\|\nabla_{H} \varphi^{\alpha}\right\|_{0, \omega}^{2}+ \\
+\sum_{\alpha=1}^{N-1} \frac{h}{3} \int_{\omega} \nabla_{H} \varphi^{\alpha} \cdot \nabla_{H} \varphi^{\alpha+1}+\frac{h}{2}\left\|\nabla_{H} \varphi^{N}\right\|_{\omega}^{2}+ \\
+\int_{0, \omega} \frac{\left(\varphi^{1}\right)^{2}}{h / 2}+\sum_{\alpha=1}^{N-1} \int_{\omega} \frac{\left(\varphi^{\alpha+1}-\varphi^{\alpha}\right)^{2}}{h}+\int_{\omega} \frac{\left(\varphi^{N}\right)^{2}}{h / 2} .
\end{gathered}
$$

To obtain the first inequality in (11), 13) and Young's inequality yield

$$
\begin{gathered}
\left\|\nabla \varphi_{h}\right\|_{0, \Omega}^{2} \leq \frac{h}{2}\left\|\nabla_{H} \varphi^{1}\right\|_{0, \omega}^{2}+\sum_{\alpha=2}^{N-1} \frac{2 h}{3}\left\|\nabla_{H} \varphi^{\alpha}\right\|_{0, \omega}^{2}+ \\
+\sum_{\alpha=1}^{N-1} \frac{h}{6}\left(\left\|\nabla_{H} \varphi^{\alpha}\right\|_{0, \omega}^{2}+\left\|\nabla_{H} \varphi^{\alpha+1}\right\|_{0, \omega}^{2}\right)+\frac{h}{2}\left\|\nabla_{H} \varphi^{N}\right\|_{0, \omega}^{2}+ \\
+\int_{\omega} \frac{\left(\varphi^{1}\right)^{2}}{h / 2}+\sum_{\alpha=1}^{N-1} \int_{\omega} \frac{\left(\varphi^{\alpha+1}-\varphi^{\alpha}\right)^{2}}{h}+\int_{\omega} \frac{\left(\varphi^{N}\right)^{2}}{h / 2} \leq\left\|\left(\mathcal{T}_{h}\right)^{-1}\left(\varphi_{h}\right)\right\|_{X_{h}}^{2} .
\end{gathered}
$$


Analogously, to obtain the first inequality in (11), again (13) and Young's inequality yield

$$
\begin{gathered}
\left\|\nabla \varphi_{h}\right\|_{0, \Omega}^{2} \geq \frac{h}{2}\left\|\nabla_{H} \varphi^{1}\right\|_{0, \omega}^{2}+\sum_{\alpha=2}^{N-1} \frac{2 h}{3}\left\|\nabla_{H} \varphi^{\alpha}\right\|_{0, \omega}^{2}+ \\
-\sum_{\alpha=1}^{N-1} \frac{h}{6}\left(\left\|\nabla_{H} \varphi^{\alpha}\right\|_{0, \omega}^{2}+\left\|\nabla_{H} \varphi^{\alpha+1}\right\|_{0, \omega}^{2}\right)+\frac{h}{2}\left\|\nabla_{H} \varphi^{N}\right\|_{0, \omega}^{2}+ \\
+\int_{\omega} \frac{\left(\varphi^{1}\right)^{2}}{h / 2}+\sum_{\alpha=1}^{N-1} \int_{\omega} \frac{\left(\varphi^{\alpha+1}-\varphi^{\alpha}\right)^{2}}{h}+\int_{\omega} \frac{\left(\varphi^{N}\right)^{2}}{h / 2} \geq \frac{1}{3}\left\|\left(\mathcal{T}_{h}\right)^{-1}\left(\varphi_{h}\right)\right\|_{X_{h}}^{2},
\end{gathered}
$$

what concludes the proof with $v_{h}=\left(\mathcal{T}_{h}\right)^{-1}\left(\varphi_{h}\right)$.

Observe that this results justifies the choice of the discrete norm 10 if $X_{h}$ is considered as an external approximation of $H_{0}^{1}(\Omega)$.

The stability of the multilayer problem $(8)$ is stated next.

Theorem 1. The form $a_{h}(\cdot, \cdot)$ satisfies the following properties:

$$
\begin{gathered}
\text { 1. } \quad a_{h}\left(v_{h}, \mathcal{T}_{h}\left(v_{h}\right)\right) \geq \frac{1}{2}\left\|v_{h}\right\|_{X_{h}}\left\|\nabla\left(\mathcal{T}_{h} v_{h}\right)\right\|_{0, \Omega} \forall v_{h} \in X_{h} ; \\
\text { 2. } \quad a_{h}\left(v_{h}, \varphi_{h}\right) \leq \sqrt{3}\left\|v_{h}\right\|_{X_{h}}\left\|\nabla \varphi_{h}\right\|_{0, \Omega}, \forall v_{h} \in X_{h}, \varphi_{h} \in Y_{h} .
\end{gathered}
$$

Proof: 1. Let $v_{h} \in X_{h}$ and $\varphi_{h} \in Y_{h}$. It holds

$$
\begin{aligned}
& \sum_{\alpha=1}^{N}\left(\nabla v_{h}^{\alpha}, \nabla \varphi_{h}\right)_{\Omega_{\alpha}}=\sum_{\alpha=1}^{N} \int_{z_{\alpha-1 / 2}}^{z_{\alpha+1 / 2}} \int_{\omega} \nabla_{H} v_{h}^{\alpha} \cdot \nabla_{H} \varphi_{h} \\
& =\int_{z_{b}}^{z_{1}} \int_{\omega} \frac{z-z_{b}}{h / 2} \nabla_{H} \varphi^{1} \cdot \nabla_{H} v_{h}^{1}+\int_{z_{N}}^{z_{s}} \int_{\omega} \frac{z_{s}-z}{h / 2} \nabla_{H} \varphi^{N} \cdot \nabla_{H} v_{h}^{N} \\
& +\sum_{\alpha=1}^{N-1} \int_{z_{\alpha}}^{z_{\alpha+1 / 2}} \int_{\omega}\left(\frac{z_{\alpha+1}-z}{h} \nabla_{H} \varphi^{\alpha}+\frac{z-z_{\alpha}}{h} \nabla_{H} \varphi^{\alpha+1}\right) \cdot \nabla_{H} v_{h}^{\alpha} \\
& +\sum_{\alpha=2}^{N} \int_{z_{\alpha-1 / 2}}^{z_{\alpha}} \int_{\omega}\left(\frac{z_{\alpha}-z}{h} \nabla_{H} \varphi^{\alpha-1}+\frac{z-z_{\alpha-1}}{h} \nabla_{H} \varphi^{\alpha}\right) \cdot \nabla_{H} v_{h}^{\alpha} \\
& =\left(\frac{3 h}{8}+\frac{h}{4}\right) \int_{\omega} \nabla_{H} v_{h}^{1} \cdot \nabla_{H} \varphi^{1}+\left(\frac{3 h}{8}+\frac{h}{4}\right) \int_{\omega} \nabla_{H} v_{h}^{N} \cdot \nabla_{H} \varphi^{N} \\
& +\frac{h}{8} \sum_{\alpha=2}^{N} \int_{\omega} \nabla_{H} v_{h}^{\alpha} \cdot \nabla_{H} \varphi^{\alpha-1}+\frac{3 h}{4} \sum_{\alpha=2}^{N-1} \int_{\omega} \nabla_{H} v_{h}^{\alpha} \cdot \nabla_{H} \varphi^{\alpha} \\
& +\frac{h}{8} \sum_{\alpha=1}^{N-1} \int_{\omega} \nabla_{H} v_{h}^{\alpha} \cdot \nabla_{H} \varphi^{\alpha+1} .
\end{aligned}
$$


Consequently, we can express $a_{h}\left(v_{h}, \varphi_{h}\right)$, as

$$
\begin{aligned}
& a_{h}\left(v_{h}, \varphi_{h}\right)=\frac{5 h}{8} \int_{\omega} \nabla_{H} v_{h}^{1} \cdot \nabla_{H} \varphi^{1}+\frac{5 h}{8} \int_{\omega} \nabla_{H} v_{h}^{N} \cdot \nabla_{H} \varphi^{N} \\
& +\frac{3 h}{4} \sum_{\alpha=2}^{N-1} \int_{\omega} \nabla_{H} v_{h}^{\alpha} \cdot \nabla_{H} \varphi^{\alpha} \\
& +\frac{h}{8} \sum_{\alpha=1}^{N-1}\left(\int_{\omega} \nabla_{H} v_{h}^{\alpha} \cdot \nabla_{H} \varphi^{\alpha+1}+\int_{\omega} \nabla_{H} v_{h}^{\alpha+1} \cdot \nabla_{H} \varphi^{\alpha}\right)+ \\
& +\int_{\omega} v_{h}^{1} \frac{\varphi^{1}}{h / 2}+\int_{\omega} v_{h}^{N} \frac{\varphi^{N}}{h / 2}+\sum_{\alpha=1}^{N-1} \int_{\omega}\left(v_{h}^{\alpha+1}-v_{h}^{\alpha}\right) \frac{\varphi^{\alpha+1}-\varphi^{\alpha}}{h} .
\end{aligned}
$$

To obtain (14), let $\varphi_{h}=\mathcal{T}_{h}\left(v_{h}\right)$. Using (17), we have

$$
\begin{aligned}
& a_{h}\left(v_{h}, \varphi_{h}\right)=\frac{5 h}{8}\left\|\nabla_{H} v_{h}^{1}\right\|_{0, \omega}^{2}+\frac{5 h}{8}\left\|\nabla_{H} v_{h}^{N}\right\|_{0, \omega}^{2} \\
& +\frac{3 h}{4} \sum_{\alpha=2}^{N-1}\left\|\nabla_{H} v_{h}^{\alpha}\right\|_{0, \omega}^{2}+\frac{h}{4} \sum_{\alpha=1}^{N-1} \int_{\omega} \nabla_{H} v_{h}^{\alpha} \cdot \nabla_{H} v_{h}^{\alpha+1} \\
& +\int_{\omega} \frac{\left(v_{h}^{1}\right)^{2}}{h / 2}+\int_{\omega} \frac{\left(v_{h}^{N}\right)^{2}}{h / 2}+\sum_{\alpha=1}^{N-1} \int_{\omega} \frac{\left(v_{h}^{\alpha+1}-v_{h}^{\alpha}\right)^{2}}{h} .
\end{aligned}
$$

By Young's inequality,

$$
\int_{\omega} \nabla_{H} v_{h}^{\alpha} \cdot \nabla_{H} v_{h}^{\alpha+1} \geq-\frac{1}{2}\left(\left\|\nabla_{H} v_{h}^{\alpha}\right\|_{0, \omega}^{2}+\left\|\nabla_{H} v_{h}^{\alpha+1}\right\|_{0, \omega}^{2}\right) .
$$

Then, from 18, it follows

$$
\begin{gathered}
a_{h}\left(v_{h}, \varphi_{h}\right) \geq \frac{h}{2} \sum_{\alpha=1}^{N}\left\|\nabla_{H} v_{h}^{\alpha}\right\|_{0, \omega}^{2}+\int_{\omega} \frac{\left(v_{h}^{1}\right)^{2}}{h / 2}+\int_{\omega} \frac{\left(v_{h}^{N}\right)^{2}}{h / 2} \\
+\sum_{\alpha=1}^{N-1} \int_{\omega} \frac{\left(v_{h}^{\alpha+1}-v_{h}^{\alpha}\right)^{2}}{h} \geq \frac{1}{2}\left\|v_{h}\right\|_{X_{h}}^{2} \geq \frac{1}{2}\left\|v_{h}\right\|_{X_{h}}\left\|\nabla \varphi_{h}\right\|_{0, \Omega} .
\end{gathered}
$$

2. Let $v_{h} \in X_{h}$ and $\varphi_{h} \in Y_{h}$, starting from (17) and using (11) and Holder's inequality, we have 


$$
\begin{aligned}
& a_{h}\left(v_{h}, \varphi_{h}\right) \leq \frac{5 h}{8}\left\|\nabla_{H} v_{h}^{1}\right\|_{0, \omega}\left\|\nabla_{H} \varphi^{1}\right\|_{0, \omega}+\frac{5 h}{8}\left\|\nabla_{H} v_{h}^{N}\right\|_{0, \omega}\left\|\nabla_{H} \varphi^{N}\right\|_{0, \omega}+ \\
& +\sum_{\alpha=2}^{N-1} \frac{3 h}{4}\left\|\nabla_{H} v_{h}^{\alpha}\right\|_{0, \omega}\left\|\nabla_{H} \varphi^{\alpha}\right\|_{0, \omega}+\sum_{\alpha=1}^{N-1} \frac{h}{8}\left\|\nabla_{H} v_{h}^{\alpha}\right\|_{0, \omega}\left\|\nabla_{H} \varphi^{\alpha+1}\right\|_{0, \omega} \\
& +\sum_{\alpha=1}^{N-1} \frac{h}{8}\left\|\nabla_{H} v_{h}^{\alpha+1}\right\|_{0, \omega}\left\|\nabla_{H} \varphi^{\alpha}\right\|_{0, \omega}+\left(\int_{\Gamma_{b}} \frac{\left(v_{h}^{1}\right)^{2}}{h / 2}\right)^{1 / 2}\left(\int_{\Gamma_{b}} \frac{\left(\varphi^{1}\right)^{2}}{h / 2}\right)^{1 / 2}+ \\
& +\sum_{\alpha=1}^{N-1}\left(\int_{\Gamma_{\alpha+\frac{1}{2}}} \frac{\left(v_{h}^{\alpha+1}-v_{h}^{\alpha}\right)^{2}}{h}\right)^{1 / 2}\left(\int_{\Gamma_{\alpha+\frac{1}{2}}} \frac{\left(\varphi^{\alpha+1}-\varphi^{\alpha}\right)^{2}}{h}\right)^{1 / 2}+ \\
& +\left(\int_{\Gamma_{s}} \frac{\left(v_{h}^{N}\right)^{2}}{h / 2}\right)^{1 / 2}\left(\int_{\Gamma_{s}} \frac{\left(\varphi^{N}\right)^{2}}{h / 2}\right)^{1 / 2} \leq \\
& \leq\left\{\frac{5 h}{8}\left\|\nabla_{H} v_{h}^{1}\right\|_{0, \omega}^{2}+\frac{5 h}{8}\left\|\nabla_{H} v_{h}^{N}\right\|_{0, \omega}^{2}+\sum_{\alpha=2}^{N-1} \frac{3 h}{4}\left\|\nabla_{H} v_{h}^{\alpha}\right\|_{0, \omega}^{2}+\right. \\
& +\sum_{\alpha=1}^{N-1} \frac{h}{8}\left\|\nabla_{H} v_{h}^{\alpha}\right\|_{0, \omega}^{2}+\sum_{\alpha=1}^{N-1} \frac{h}{8}\left\|\nabla_{H} v_{h}^{\alpha+1}\right\|_{0, \omega}^{2}+\sum_{\alpha=1}^{N-1} \int_{\Gamma_{\alpha+\frac{1}{2}}} \frac{\left(v_{h}^{\alpha+1}-v_{h}^{\alpha}\right)^{2}}{h}+ \\
& \left.+\int_{\Gamma_{b}} \frac{\left(v_{h}^{1}\right)^{2}}{h / 2}+\int_{\Gamma_{s}} \frac{\left(v_{h}^{N}\right)^{2}}{h / 2}\right\}^{1 / 2} \cdot\left\{\frac{5 h}{8}\left\|\nabla_{H} \varphi^{1}\right\|_{0, \omega}^{2}+\frac{5 h}{8}\left\|\nabla_{H} \varphi^{N}\right\|_{0, \omega}^{2}+\right. \\
& +\sum_{\alpha=2}^{N-1} \frac{3 h}{4}\left\|\nabla_{H} \varphi^{\alpha}\right\|_{0, \omega}^{2}+\sum_{\alpha=1}^{N-1} \frac{h}{8}\left\|\nabla_{H} \varphi^{\alpha}\right\|_{0, \omega}^{2}+\sum_{\alpha=1}^{N-1} \frac{h}{8}\left\|\nabla_{H} \varphi^{\alpha+1}\right\|_{0, \omega}^{2}+ \\
& \left.+\sum_{\alpha=1}^{N-1} \int_{\Gamma_{\alpha+\frac{1}{2}}} \frac{\left(\varphi^{\alpha+1}-\varphi^{\alpha}\right)^{2}}{h}+\int_{\Gamma_{b}} \frac{\left(\varphi^{1}\right)^{2}}{h / 2}+\int_{\Gamma_{s}} \frac{\left(\varphi^{N}\right)^{2}}{h / 2}\right\}^{1 / 2} \leq \\
& \leq
\end{aligned}
$$

As a conclusion we deduce

Corollary 2. The form $a_{h}$ satisfies the inf-sup condition

$$
\inf _{v_{h} \in X_{h}} \sup _{\varphi_{h} \in Y_{h}} \frac{a_{h}\left(v_{h}, \varphi_{h}\right)}{\left\|\nabla \varphi_{h}\right\|_{0, \Omega}\left\|v_{h}\right\|_{X_{h}}} \geq \frac{1}{2} .
$$

Proof: It is a direct consequence of estimate (14).

This implies the well posedness of problem (8): 
Corollary 3. The multilayer problem (8) admits a unique solution $v_{h} \in X_{h}$ that satisfies the estimate

$$
\left\|v_{h}\right\|_{X_{h}} \leq 2\|f\|_{H^{-1}(\Omega)} .
$$

Proof: The form $a_{h}$ is stable by Theorem 1 and satisfies the inf-sup condition (20). Also, (14) implies that if $a_{h}\left(v_{h}, \varphi_{h}\right)=0$ for all $v_{h} \in X_{h}$, for some $\varphi_{h} \in Y_{h}$, then necessarily $\varphi_{h}=0$. Consequently the hypotheses of the Banach-NecasBabuska Theorem hold (Cf. [14]). This ensures that problem (8) admits a unique solution that depends continuously on the data $f$. The constant 2 in estimate 21) also follows from (14).

\subsection{Convergence analysis}

In this section we prove a convergence result for general solutions of problem (2), so as optimal order error estimates for smooth solutions. For that, let us construct an interpolation operator on $X_{h}$ for functions defined on $\Omega$. Let $\mathcal{P}_{h}$ be the prismatic grid of $\Omega$ constructed by vertical displacements of the grid $\mathcal{T}_{k}$, located at the nodes $z_{b}, z_{1}, z_{2} \cdots, z_{N-1}, z_{N}, z_{s}$. The geometric elements of $\mathcal{P}_{h}$ are constructed as $P=K \times I_{j}$, where $I_{1}=\left[z_{b}, z_{1}\right], I_{j}=\left[z_{j-1}, z_{j}\right], j=2, \cdots, N$; $I_{N+1}=\left[z_{N}, z_{s}\right]$ and $K \in \mathcal{T}_{k}$. Note that $Y_{h}$ is the prismatic finite element space defined by

$$
Y_{h}=\left\{w \in C^{0}(\Omega) \mid w_{\left.\right|_{P}} \in R_{l}(K) \times P_{1}\left(I_{j}\right), \forall P=K \times I_{j} \in \mathcal{P}_{h}\right\} \cap H_{0}^{1}(\Omega) .
$$

If $\left\{x_{i}\right\}_{i \in \mathcal{I}}$ is the set of Lagrange interpolation nodes for $V_{0, k}(\omega)$, for some set of indices $\mathcal{I}$, then the set of Lagrange interpolation nodes for $Y_{h}$ is $\left\{r_{i, j}=\left(x_{i}, z_{j}\right) \mid i \in \mathcal{I}, j=1, \cdots, N\right\}$. Following Bernardi et al. [15], we introduce a nodal Lagrange interpolation operator $\Pi_{k}: L^{2}(\Omega) \mapsto Y_{h}$, of the form

$$
\left(\Pi_{k} w\right)(x, z)=\sum_{i \in \mathcal{I}} \sum_{j=1}^{N} \bar{w}_{i, j} \alpha_{i, j}(x, z),
$$

where the $\alpha_{i, j}$ are the nodal Lagrange interpolation basis functions on $V_{0, k}(\omega)$ corresponding interpolation nodes $r_{i, j}$, and the $\bar{w}_{i, j}$ are averaged value of $w$ on an element to which the node $r_{i, j}$ belongs. 
The operator $\Pi_{k}$ satisfies the standard finite element interpolation error estimates,

$$
\begin{gathered}
\left\|w-\Pi_{k} w\right\|_{0, \Omega} \leq \hat{C}\left(h+k^{l}\right)\|w\|_{l, \Omega}, \text { if } w \in H^{l}(\Omega), \\
\left\|w-\Pi_{k} w\right\|_{1, \Omega} \leq \hat{C}\left(h+k^{l}\right)\|w\|_{l+1, \Omega}, \quad \text { if } w \in H^{l+1}(\Omega) .
\end{gathered}
$$

We shall assume that the operator $\Pi_{k}$ also is defined componentwise for vector functions, without change of notations. We next define an interpolation operator $\Pi_{h}: H_{0}^{1}(\Omega) \longrightarrow X_{h}$ that transforms $v \in H_{0}^{1}(\Omega)$ into $\Pi_{h} v \in X_{h}$, defined by $\left.\left(\Pi_{h} v\right)\right|_{\Omega_{\alpha}}=v_{k}^{\alpha} \in V_{0, k}(\omega), \quad \forall \alpha=1,2, \cdots, N$, with

$$
v_{k}^{\alpha}(x)=\frac{1}{h} \int_{z_{\alpha-\frac{1}{2}}}^{z_{\alpha+\frac{1}{2}}} \Pi_{k} v(x, z) d z \quad x \in w \text { a. e.. }
$$

We at first obtain the optimal order error estimate

Theorem 4. Assume that the weak solution of (2) satisfies $v \in H^{l+1}(\Omega) \cap$ $H_{0}^{1}(\Omega)$, with $l \geq 1$. Then, the solution $v_{h} \in X_{h}$ of the discretization (8) verifies

$$
\left\|\Pi_{h} v-v_{h}\right\|_{X_{h}} \leq C\|v\|_{l+1, \Omega}\left(h+k^{l}\right)
$$

for some constant $C>0$ a constant.

Proof: Define the consistency error $\varepsilon_{h} \in Y_{h}^{\prime}$ by

$$
<\varepsilon_{h}, \varphi_{h}>=a_{h}\left(\Pi_{h} v-v_{h}, \varphi_{h}\right), \quad \forall \varphi_{h} \in Y_{h}
$$

It is sufficient show that, there exits a constant $C^{\prime}>0$ such that

$$
\left|<\varepsilon_{h}, \varphi_{h}>\right| \leq C^{\prime}\|v\|_{l+1, \Omega}\left\|\nabla \varphi_{h}\right\|_{0, \Omega}\left(h+k^{l}\right), \quad \forall \varphi_{h} \in Y_{h} .
$$

Indeed, by the inf-sup condition, we have

$$
\begin{gathered}
\frac{1}{2}\left\|\Pi_{h} v-v_{h}\right\|_{X_{h}} \leq \sup _{\varphi_{h} \in Y_{h}} \frac{a_{h}\left(\Pi_{h} v-v_{h}, \varphi_{h}\right)}{\left\|\nabla \varphi_{h}\right\|_{0, \Omega}}= \\
=\sup _{\varphi_{h} \in Y_{h}} \frac{<\varepsilon_{h}, \varphi_{h}>}{\left\|\nabla \varphi_{h}\right\|_{0, \Omega}} \leq C^{\prime}\|v\|_{l+1, \Omega}\left(h+k^{l}\right),
\end{gathered}
$$

and we obtain the error estimate with $C=2 C^{\prime}$. 
To prove 26), notice that $a_{h}\left(v_{h}, \varphi_{h}\right)=L\left(\varphi_{h}\right)=a\left(v, \varphi_{h}\right)$, and then

$$
\begin{gathered}
<\varepsilon_{h}, \varphi_{h}>=a_{h}\left(\Pi_{h} v-v_{h}, \varphi_{h}\right)=a_{h}\left(\Pi_{h} v, \varphi_{h}\right)-a\left(v, \varphi_{h}\right)= \\
=\sum_{\alpha=1}^{N} \int_{\Omega_{\alpha}} \nabla\left(v^{\alpha}-v\right) \nabla \varphi_{h}+\int_{\Gamma_{b}} v^{1} \partial_{z} \varphi_{h}+\sum_{\alpha=1}^{N-1} \int_{\Gamma_{\alpha+\frac{1}{2}}}\left(v^{\alpha+1}-v^{\alpha}\right) \partial_{z} \varphi_{h}+ \\
-\int_{\Gamma_{s}} v^{N} \partial_{z} \varphi_{h}=\sum_{\alpha=1}^{N} \int_{\Omega_{\alpha}} \nabla_{H}\left(v^{\alpha}-v\right) \nabla_{H} \varphi_{h}-\sum_{\alpha=1}^{N} \int_{\Omega_{\alpha}} \partial_{z} v \partial_{z} \varphi_{h}+ \\
+\int_{\omega} \int_{z_{b}}^{z_{1}} \frac{v^{1}}{h / 2} \partial_{z} \varphi_{h}+\sum_{\alpha=1}^{N-1} \int_{\omega} \int_{z_{\alpha}}^{z_{\alpha+1}} \frac{v^{\alpha+1}-v^{\alpha}}{h} \partial_{z} \varphi_{h}+\int_{\omega} \int_{z_{N}}^{z_{s}} \frac{-v^{N}}{h / 2} \partial_{z} \varphi_{h}= \\
=\sum_{\alpha=1}^{N} \int_{\Omega_{\alpha}} \nabla_{H}\left(\Pi_{h} v-v\right) \nabla_{H} \varphi_{h}-\sum_{\alpha=1}^{N} \int_{\Omega_{\alpha}} \partial_{z} v \partial_{z} \varphi_{h}+\int_{\omega} \int_{z_{b}}^{z_{1}} \partial_{z}\left(\mathcal{T}_{h}\left(\Pi_{h} v\right)\right) \partial_{z} \varphi_{h}+ \\
+\sum_{\alpha=1}^{N-1} \int_{\omega} \int_{z_{\alpha}}^{z_{\alpha+1}} \partial_{z}\left(\mathcal{T}_{h}\left(\Pi_{h} v\right)\right) \partial_{z} \varphi_{h}+\int_{\omega} \int_{z_{N}}^{z_{s}} \partial_{z}\left(\mathcal{T}_{h}\left(\Pi_{h} v\right)\right) \partial_{z} \varphi_{h} .
\end{gathered}
$$

Then

$$
<\varepsilon_{h}, \varphi_{h}>=\int_{\Omega} \nabla_{H}\left(\Pi_{h} v-v\right) \cdot \nabla_{H} \varphi_{h}+\int_{\Omega} \partial_{z}\left(\mathcal{T}_{h}\left(\Pi_{h} v\right)-v\right) \partial_{z} \varphi_{h} .
$$

To estimate the first term in (27), we have

$$
\left|\int_{\Omega} \nabla_{H}\left(\Pi_{h} v-v\right) \cdot \nabla_{H} \varphi_{h}\right| \leq\left\|\nabla_{H}\left(\Pi_{h} v-v\right)\right\|_{0, \Omega}\left\|\nabla_{H} \varphi_{h}\right\|_{0, \Omega} .
$$

Now we add $\pm\left(\nabla_{H} v\right)(x, \hat{z})$ to the integral, use Holder's and Minkowski's inequalities and apply that if $v \in H^{2}(\Omega)$, it holds

$$
\left(\nabla_{H} v\right)(x, \hat{z})=\left(\nabla_{H} v\right)(x, z)+\int_{\hat{z}}^{z} \partial_{z}\left(\nabla_{H} v\right)(x, s) d s
$$

for $z, \hat{z} \in\left(z_{\alpha-\frac{1}{2}}, z_{\alpha+\frac{1}{2}}\right)$, a. e. for $x \in \omega$. Then,

$$
\begin{aligned}
& \left\|\nabla_{H}\left(\Pi_{h} v\right)-\nabla_{H} v\right\|_{0, \Omega}= \\
& =\left(\sum_{\alpha=1}^{N} \int_{\Omega_{\alpha}}\left|\frac{1}{h} \int_{z_{\alpha-\frac{1}{2}}}^{z_{\alpha+\frac{1}{2}}}\left(\nabla_{H}\left(\Pi_{k} v\right)(x, \hat{z})-\left(\nabla_{H} v\right)(x, z)\right) d \hat{z}\right|^{2} d x d z\right)^{1 / 2} \leq \\
& \leq\left(\frac{1}{h} \sum_{\alpha=1}^{N} \int_{\Omega_{\alpha}} \int_{z_{\alpha-\frac{1}{2}}}^{z_{\alpha+\frac{1}{2}}}\left|\left(\nabla_{H}\left(\Pi_{k} v\right)(x, \hat{z})-\left(\nabla_{H} v\right)(x, z)\right)\right|^{2} d \hat{z} d x d z\right)^{1 / 2} \leq
\end{aligned}
$$




$$
\begin{aligned}
& \leq\left(\frac{1}{h} \sum_{\alpha=1}^{N} \int_{\Omega_{\alpha}} \int_{z_{\alpha-\frac{1}{2}}}^{z_{\alpha+\frac{1}{2}}}\left|\left(\nabla_{H}\left(\Pi_{k} v\right)(x, \hat{z})-\left(\nabla_{H} v\right)(x, \hat{z})\right)\right|^{2} d \hat{z} d x d z\right)^{1 / 2}+ \\
& +\left(\frac{1}{h} \sum_{\alpha=1}^{N} \int_{\Omega_{\alpha}} \int_{z_{\alpha-\frac{1}{2}}}^{z_{\alpha+\frac{1}{2}}}\left|\left(\left(\nabla_{H} v\right)(x, \hat{z})-\left(\nabla_{H} v\right)(x, z)\right)\right|^{2} d \hat{z} d x d z\right)^{1 / 2}= \\
& =\left(\sum_{\alpha=1}^{N} \int_{\Omega_{\alpha}}\left|\left(\nabla_{H}\left(\Pi_{k} v\right)(x, \hat{z})-\left(\nabla_{H} v\right)(x, \hat{z})\right)\right|^{2} d x d \hat{z}\right)^{1 / 2}+ \\
& +\left(\frac{1}{h} \sum_{\alpha=1}^{N} \int_{\Omega_{\alpha}} \int_{z_{\alpha-\frac{1}{2}}}^{z_{\alpha+\frac{1}{2}}}\left|\int_{\hat{z}}^{z} \partial_{z}\left(\nabla_{H} v\right)(x, s) d s\right|^{2} d \hat{z} d x d z\right)^{1 / 2} \leq \\
& \leq\left\|\nabla_{H}\left(\Pi_{k} v-v\right)\right\|_{0, \Omega}+h\left\|\partial_{z}\left(\nabla_{H} v\right)\right\|_{0, \Omega} \leq \\
& \leq \hat{C}\left(h+k^{l}\right)\|v\|_{l+1, \Omega}+h\left\|\partial_{z}\left(\nabla_{H} v\right)\right\|_{0, \Omega} .
\end{aligned}
$$

where the last inequality follows from (23).

To estimate the term in $\partial_{z}$ in (27), we have

$$
\left|\int_{\Omega} \partial_{z}\left(\mathcal{T}_{h}\left(\Pi_{h} v\right)-v\right) \partial_{z} \varphi_{h}\right| \leq\left\|\partial_{z}\left(\mathcal{T}_{h}\left(\Pi_{h} v\right)-v\right)\right\|_{0, \Omega}\left\|\partial_{z} \varphi_{h}\right\|_{0, \Omega} .
$$

Observe that the composed operator $\mathcal{T}_{h} \circ \Pi_{h}$ is a Clément interpolation operator (cf. [16]), so as $v \in H^{2}(\Omega)$, it holds

$$
\| \partial_{z}\left(\mathcal{T}_{h}\left(\Pi_{h} v\right)-\partial_{z} v\left\|_{0, \Omega} \leq \hat{C}^{\prime}\right\| v \|_{2, \Omega} h\right.
$$

Then, from 28 and 29, we obtain 26, with $C^{\prime}=\hat{C}^{\prime}+\hat{C}+1$.

Observation 2. The overall order of the discretization (8) is then one. Thus, to minimize the number of degrees of freedom the best choice is $l=1$ and $k=h$.

We now can deduce the general convergence result,

Corollary 5. Let $v \in H_{0}^{1}(\Omega)$ the solution of problem (2). Then it holds

$$
\lim _{h \rightarrow 0}\left\|\Pi_{h} v-v_{h}\right\|_{X_{h}}=0
$$

where $v_{h}$ is the solution of the multilayer discretization (8). 
Proof: As in the proof of Theorem 4 , it holds

$$
\begin{aligned}
\frac{1}{2}\left\|\Pi_{h} v-v_{h}\right\|_{X_{h}} & \leq \sup _{\varphi_{h} \in Y_{h}} \frac{\left\langle\varepsilon_{h}, \varphi_{h}>\right.}{\left\|\nabla \varphi_{h}\right\|_{0, \Omega}} \\
& \leq\left\|\nabla_{H}\left(\Pi_{h} v-v\right)\right\|_{0, \Omega}+\left\|\partial_{z}\left(\left(\mathcal{T}_{h} \circ \Pi_{h}\right) v-v\right)\right\|_{0, \Omega} .
\end{aligned}
$$

Operators $\Pi_{h}$ and $\mathcal{T}_{h} \circ \Pi_{h}$ are stable, in the sense that

$$
\begin{gathered}
\left\|\nabla_{H}\left(\Pi_{h} w\right)\right\|_{0, \Omega} \leq C\|w\|_{1, \Omega}, \forall w \in H^{1}(\Omega), \\
\left\|\partial_{z}\left(\left(\mathcal{T}_{h} \circ \Pi_{h}\right) w\right)\right\|_{0, \Omega} \leq C\left\|\partial_{z} w\right\|_{0, \Omega}, \quad \forall w \in H^{1}(\Omega) .
\end{gathered}
$$

Indeed, is straightforward to prove that $\left\|\nabla_{H}\left(\Pi_{h} w\right)\right\|_{0, \Omega} \leq\left\|\Pi_{k} w\right\|_{1, \Omega}$ and then the first estimate follows as it because $\Pi_{k}$ is stable in $H^{1}(\Omega)$ norm. The second one is a standard property of the Clément interpolation operator (cf. [16]). Then a standard argument, using the density of $\mathcal{D}(\Omega)$ in $H_{0}^{1}(\Omega)$, proves that the r. h. s. of (31) tends to zero as $h \rightarrow 0$.

\section{Extension to domains with non-flat upper boundary}

In this section we extend the multilayer discretization of the Poisson problem (1) to domains with non-flat upper boundary, that arise in geophysical flows. Concretely, we consider domains $\hat{\Omega}$ that can be obtained as vertical transformations of the domain $\Omega=\omega \times(0,1)$ (that we call in this section "reference" domain) by a change of variables of the form:

$$
\hat{z}=z \eta(x), \quad \hat{x}=x,
$$

with $x \in \omega \subset \mathbb{R}^{2}, z \in(0,1)$ and where $\eta$ is an smooth and strictly positive function defined in $\omega$ (the surface equations are thus $z=\eta(x, y)$ ). We assume that $\eta \in W^{1, \infty}(\omega)$ is a function such that

$$
\eta(x) \geq \eta_{0}>0 \text { and }\left\|\nabla_{H} \eta\right\|_{\infty, \Omega}<1, \forall x \in \omega \text { uniformly }
$$

for some constant $\eta_{0}$. Note that he restriction in 33 on the gradient of $\eta$ is consistent with the need of having $\eta>0$ on $\omega$. 
We intend to solve the Poisson problem in $\hat{\Omega}$ :

$$
\left\{\begin{aligned}
-\Delta \hat{v} & =\hat{f} \quad \text { in } \quad \hat{\Omega} \\
\hat{v} & =0 \text { on } \partial \hat{\Omega} .
\end{aligned}\right.
$$

To do it, we use the change of variables 32 , to transform problem 34 in the following elliptic problem in the reference domain $\Omega$ in variational form: Find $v \in H_{0}^{1}(\Omega)$ such that

$$
a_{K}(v, \varphi)=L(\varphi), \quad \forall \varphi \in H_{0}^{1}(\Omega),
$$

with $a_{K}(v, \varphi)=\int_{\Omega} \eta(\nabla \varphi)^{T} K \nabla v$ and $L(\varphi)=<f, \varphi>_{\Omega}$, where $f(x, z)=$ $\hat{f}(x, \hat{z})$ and $K$ is a symmetric and positive definite matrix given by

$$
K=\left(\begin{array}{cc}
K_{H H} & K_{H 3} \\
K_{3 H} & K_{33}
\end{array}\right)
$$

where $K_{H H}=I d_{H}$ with $I d_{H}$ the $2 \times 2$ identity matrix, $K_{H 3}=-\frac{z}{\eta} \nabla_{H} \eta$, $K_{3 H}=K_{H 3}^{T}$ and $K_{33}=\frac{1}{\eta^{2}}+\left|K_{H 3}\right|^{2}$

Here our objective is to deduce a multilayer approach of problem (34).

\subsection{Multilayer discretization}

To obtain our new multilayer system, we consider the same vertical decomposition of domain $\Omega$ and the same definitions and notations as those used in Section 2 .

Similarly to the definition of the form $a_{h}$ by (9), we propose the following multilayer discretization of problem (34):

$$
\left\{\begin{array}{l}
\text { Find } \quad v_{h} \in X_{h}, \quad \text { such that } \\
a_{K, h}\left(v_{h}, \varphi_{h}\right)=L\left(\varphi_{h}\right), \quad \forall \varphi_{h} \in Y_{h},
\end{array}\right.
$$

where, the bilinear form $a_{K, h}(\cdot, \cdot)$ is given by

$$
\begin{aligned}
& a_{K, h}\left(v_{h}, \varphi_{h}\right)=\int_{\Omega} \eta\left[\left(\nabla_{H} v_{h}\right)^{T} \nabla_{H} \varphi_{h}+\partial_{z}\left(\mathcal{T}_{h}\left(v_{h}\right)\right) K_{3 H} \nabla_{H} \varphi_{h}\right]+ \\
& +\int_{\Omega} \eta\left[\left(\nabla_{H} v_{h}\right)^{T} K_{3 H}^{T} \partial_{z} \varphi_{h}+\partial_{z}\left(\mathcal{T}_{h}\left(v_{h}\right)\right) K_{33} \partial_{z} \varphi_{h}\right] .
\end{aligned}
$$




\subsection{Analysis of the multilayer discretization}

We now study some properties of the multilayer problem (36). The stability of the multilayer discretization is achieved, under some restrictions on the gradient of function $\eta$, as follows:

Theorem 6. We assume that function $\eta$ satisfies 33). Then, the bilinear form $a_{K, h}(\cdot, \cdot)$ satisfies the following properties:

1. There exists a constant $C_{1, K}>0$ that depends on $\eta$, such that

$$
a_{K, h}\left(v_{h}, \mathcal{T}_{h}\left(v_{h}\right)\right) \geq C_{1, K}\left\|\nabla \mathcal{T}_{h}\left(v_{h}\right)\right\|_{0, \Omega}\left\|v_{h}\right\|_{X_{h}}, \quad \forall v_{h} \in X_{h} .
$$

2. There exists a constant $C_{2, K}>0$ that depends on $\eta$, such that

$$
a_{K, h}\left(v_{h}, \varphi_{h}\right) \leq C_{2, K}\left\|\nabla \varphi_{h}\right\|_{0, \Omega}\left\|v_{h}\right\|_{X_{h}}, \forall v_{h} \in X_{h}, \forall \varphi_{h} \in Y_{h} .
$$

\section{Proof:}

1. To obtain (38), let $v_{h} \in X_{h}$ and $\varphi_{h}=\mathcal{T}_{h}\left(v_{h}\right)$, then from (37) we have

$$
\begin{aligned}
& a_{K, h}\left(v_{h}, \mathcal{T}_{h}\left(v_{h}\right)\right)=\int_{\Omega} \eta\left[\left(\nabla_{H} v_{h}\right)^{T} \nabla_{H}\left(\mathcal{T}_{h}\left(v_{h}\right)\right)+K_{33}\left|\partial_{z} \mathcal{T}_{h}\left(v_{h}\right)\right|^{2}\right]+ \\
& +\int_{\Omega} \eta\left[\partial_{z} \mathcal{T}_{h}\left(v_{h}\right) K_{3 H} \nabla_{H}\left(\mathcal{T}_{h}\left(v_{h}\right)+v_{h}\right)\right] .
\end{aligned}
$$

Now, using that $\eta>0$, Young's inequality $\left(a b \geq-\frac{1}{2}\left(\epsilon a^{2}+\frac{1}{\epsilon} b^{2}\right)\right)$ for the last integral and the estimates within the proof of 111) in Lemma 1 for the integrals with $\nabla_{H} v_{h}$ or $\nabla_{H}\left(\mathcal{T}_{h}\left(v_{h}\right)\right)$, we obtain

$$
\begin{aligned}
& a_{K, h}\left(v_{h}, \mathcal{T}_{h}\left(v_{h}\right)\right) \geq \int_{\Omega} \eta\left[\frac{1}{2}\left|\nabla_{H} v_{h}\right|^{2}+K_{33}\left|\partial_{z} \mathcal{T}_{h}\left(v_{h}\right)\right|^{2}\right]+ \\
& -\frac{\epsilon}{2} \int_{\Omega} \eta\left|K_{3 H}\right|^{2}\left|\partial_{z} \mathcal{T}_{h}\left(v_{h}\right)\right|^{2}-\frac{1}{\epsilon} \int_{\Omega} \eta\left(\left|\nabla_{H}\left(\mathcal{T}_{h}\left(v_{h}\right)\right)\right|^{2}+\left|\nabla_{H} v_{h}\right|^{2}\right) \geq \\
& \geq\left(\frac{1}{2}-\frac{2}{\epsilon}\right) \int_{\Omega} \eta\left|\nabla_{H} v_{h}\right|^{2}+\int_{\Omega} \eta\left(K_{33}-\frac{\epsilon}{2}\left|K_{3 H}\right|^{2}\right)\left|\partial_{z} \mathcal{T}_{h}\left(v_{H}\right)\right|^{2} .
\end{aligned}
$$

Then, if we take $\varepsilon=4(1+\beta)$ with $\beta>0$ and we use that $\left\|\nabla_{H} \eta\right\|_{\infty, \Omega}<1$, we have

$$
\begin{aligned}
& a_{K, h}\left(v_{h}, \mathcal{T}_{h}\left(v_{h}\right)\right) \geq \int_{\Omega}\left(\frac{1-(1+2 \beta) z^{2}\left(\left|\nabla_{H} \eta\right|^{2}\right)}{\eta}\right)\left|\partial_{z} \mathcal{T}_{h}\left(v_{h}\right)\right|^{2}+ \\
& +\frac{\beta}{2(1+\beta)} \int_{\Omega} \eta\left|\nabla_{H} v_{h}\right|^{2} \geq C_{1, K}\left\|v_{h}\right\|_{X_{h}}^{2} \geq C_{1, K}\left\|\nabla\left(\mathcal{T}_{h}\left(v_{h}\right)\right)\right\|_{0, \Omega}\left\|v_{h}\right\|_{X_{h}} .
\end{aligned}
$$


with $C_{1, K}=\min \left\{\frac{\beta \eta_{0}}{2(1+\beta)}, \frac{1-(1+2 \beta)\left\|\nabla_{H} \eta\right\|_{\infty, \Omega}^{2}}{\|\eta\|_{\infty, \Omega}}\right\}$, where for the last inequality we use 111.

2. Let $v_{h} \in X_{h}$ and $\varphi_{h} \in Y_{h}$, then as $\eta>0$, 11 and Holders inequality, we have

$$
\begin{gathered}
a_{K, h}\left(v_{h}, \varphi_{h}\right) \leq\left(\int_{\Omega} \eta\left|\nabla_{H} v_{h}\right|^{2}\right)^{1 / 2}\left(\int_{\Omega} \eta\left|\nabla_{H} \varphi_{h}\right|^{2}\right)^{1 / 2}+ \\
+\left(\int_{\Omega} \eta\left|K_{3 H}\right|^{2}\left|\partial_{z} \mathcal{T}_{h}\left(v_{h}\right)\right|^{2}\right)^{1 / 2}\left(\int_{\Omega} \eta\left|\nabla_{H} \varphi_{h}\right|^{2}\right)^{1 / 2}+\left(\int_{\Omega} \eta\left|\nabla_{H} v_{h}\right|^{2}\right)^{1 / 2} \cdot \\
\cdot\left(\int_{\Omega} \eta\left|K_{3 H}\right|^{2}\left|\partial_{z} \varphi_{h}\right|^{2}\right)^{1 / 2}+\left(\int_{\Omega} \eta K_{33}\left|\partial_{z} \mathcal{T}_{h}\left(v_{H}\right)\right|^{2}\right)^{1 / 2}\left(\int_{\Omega} \eta K_{33}\left|\partial_{z} \varphi_{h}\right|^{2}\right)^{1 / 2} \leq \\
\leq\left\{2 \int_{\Omega} \eta\left|\nabla_{H} v_{h}\right|^{2}+\int_{\Omega} \eta\left(\left|K_{3 H}\right|^{2}+K_{33}\right)\left|\partial_{z} \mathcal{T}_{h}\left(v_{h}\right)\right|^{2}\right\}^{1 / 2} \cdot \\
\cdot\left\{2 \int_{\Omega} \eta\left|\nabla_{H} \varphi_{h}\right|^{2}+\int_{\Omega} \eta\left(\left|K_{3 H}\right|^{2}+K_{33}\right)\left|\partial_{z} \varphi_{h}\right|^{2}\right\}^{1 / 2} \leq \\
\leq C_{K}\left\|v_{h}\right\|\left\|_{X_{h}}\right\|\left(\mathcal{T}_{h}\right)^{-1}\left(\varphi_{h}\right)\left\|_{X_{h}} \leq \sqrt{3} C_{K}\right\| v_{h}\left\|_{X_{h}}\right\| \nabla \varphi_{h} \|_{0, \Omega} .
\end{gathered}
$$

Thus, we obtain 39 with $C_{2, K}=\sqrt{3} C_{K}$ where $C_{K}=\min \left\{2\|\eta\|_{\infty, \Omega}, \frac{1+2\left\|\nabla_{H} \eta\right\|_{\infty, \Omega}^{2}}{\eta_{0}}\right\}$.

As consequence of this theorem we deduce the following well posedness result for the multilayer problem 36 :

Corollary 7. We assume that function $\eta$ satisfies (33), then the multilayer problem (36) admits a unique solution $v_{h} \in X_{h}$ that satisfies the estimate

$$
\left\|v_{h}\right\|_{X_{h}} \leq C_{3, K}\|f\|_{H^{-1}(\Omega)}
$$

where $C_{3, K}>0$ is a constant that depends on $\eta$.

The convergence of the multilayer discretization 36 is stated as follows:

Theorem 8. We assume that $\eta$ verifies (33) and that the weak solution of (35) satisfies $v \in H^{l+1}(\Omega) \cap H_{0}^{1}(\Omega)$, with $l \geq 1$. Then, the solution $v_{h} \in X_{h}$ of the discrete problem (36) verifies the estimate

$$
\left\|\Pi_{h} v-v_{h}\right\|_{X_{h}} \leq\left(h+k^{l}\right) C_{4, K}\|v\|_{l+1, \Omega}
$$


with $C_{4, K}$ a constant that depends on $\eta$ and $\Pi_{h}$ the operator defined by (24).

Moreover, if $v \in H_{0}^{1}(\Omega)$, then, it holds

$$
\lim _{h \rightarrow 0}\left\|\Pi_{h} v-v_{h}\right\|_{X_{h}}=0
$$

The proof of this result is an extension of that of Theorem 4 and Corollary 5 by standard techniques, we omit it for brevity.

\section{Neumann boundary conditions}

In this section we extend the multilayer discretization to the Poisson problem with Dirichlet boundary conditions (1) to the following Poisson problem with Dirichlet-Neumann boundary conditions:

$$
\left\{\begin{array}{ccccc}
-\Delta v & = & f & \text { in } & \Omega, \\
v & = & 0 & \text { on } & \Gamma_{b} \cup \Gamma_{l}, \\
\partial_{n} v & = & g & \text { on } & \Gamma_{s} .
\end{array}\right.
$$

The variational form of this problem is: Given $f \in H^{-1}(\Omega)$ and $g \in H^{-1 / 2}\left(\Gamma_{s}\right)$, find $v \in H_{b l}^{1}(\Omega)$ such that

$$
a(v, \hat{\varphi})=\hat{L}(\hat{\varphi}), \quad \forall \hat{\varphi} \in H_{b l}^{1}(\Omega),
$$

with

$$
a(v, \hat{\varphi})=\int_{\Omega} \nabla v \nabla \hat{\varphi} d \Omega, \quad \text { and } \quad \hat{L}(\hat{\varphi})=<f, \hat{\varphi}>_{\Omega}+\int_{\Gamma_{s}} g \hat{\varphi},
$$

where $H_{b l}^{1}(\Omega)=\left\{v \in H^{1}(\Omega) / v_{\left.\right|_{\Gamma_{b}} \cup \Gamma_{l}}=0\right\}$. Here we consider the same space of semi-discrete solutions in the vertical direction as in Section 2 , that we endow with the following $H_{b l}^{1}(\Omega)$ discrete norm:

$$
\begin{aligned}
\left\|v_{h}\right\|_{X_{h}}= & \left(h \sum_{\alpha=1}^{N}\left\|\nabla v_{h}^{\alpha}\right\|_{0, \omega}^{2}+\sum_{\alpha=1}^{N-2} h\left\|\frac{v_{h}^{\alpha+1}-v_{h}^{\alpha}}{h}\right\|_{0, \omega}^{2}\right. \\
& \left.+\frac{h}{2}\left\|\frac{v_{h}^{1}}{h / 2}\right\|_{0, \omega}^{2}+\frac{3 h}{2}\left\|\frac{v_{h}^{N}-v_{h}^{N-1}}{3 h / 2}\right\|_{0, \omega}^{2}\right)^{1 / 2} .
\end{aligned}
$$




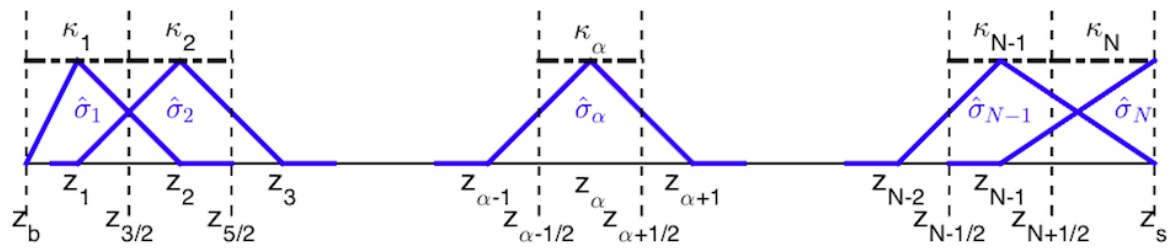

Figure 3: Basis function on $X_{h}$ and $\hat{Y}_{h}$

This new discrete norm is motivated by the following new semi-discrete test functions space,

$$
\hat{Y}_{h}=\left\{\hat{\varphi}_{h} \in L^{2}(\Omega) / \hat{\varphi}_{h}=\sum_{\alpha=1}^{N} \hat{\varphi}^{\alpha} \otimes \hat{\sigma}_{\alpha}, \text { with } \hat{\varphi}^{\alpha} \in V_{0, k}(\omega)\right\},
$$

where $\hat{\sigma}_{i}=\sigma_{i}$, for all $i=1, \ldots, N-2$, and $\hat{\sigma}_{N-1}, \hat{\sigma}_{N}$ are piecewise affine $1 \mathrm{D}$ functions on the intervals $\left[z_{N-2}, z_{N-1}\right],\left[z_{N-1}, z_{s}\right]$, respectively, that satisfy $\hat{\sigma}_{N-1}\left(z_{j}\right)=\delta_{(N-1) j}$ and $\hat{\sigma}_{N}\left(z_{j}\right)=0$, for all $j=1,2, \ldots, N-1 ; \hat{\sigma}_{N-1}\left(z_{b}\right)=$ $\hat{\sigma}_{N-1}\left(z_{s}\right)=\hat{\sigma}_{N}\left(z_{b}\right)=0$ and $\hat{\sigma}_{N}\left(z_{s}\right)=1$ as we see in Figure 3 . Note that $\hat{Y}_{h}$ is a subspace of $H_{b l}^{1}(\Omega)$ such that $\operatorname{dim}\left(\hat{Y}_{h}\right)=\operatorname{dim}\left(X_{h}\right)$ and the interval $\left[z_{N-1}, z_{s}\right]$ has length $3 h / 2$.

We look for an approximation of the solution of 42 of the form $v \simeq v_{h}+g_{h}$ with $g_{h}=g_{k}\left(z-z_{s}\right) \kappa_{N}$ and $v_{h}$ is the solution of the following multilayer discretization of 42 :

$$
\left\{\begin{array}{l}
\text { Find } v_{h} \in X_{h}, \quad \text { such that } \\
\hat{a}_{h}\left(v_{h}, \hat{\varphi}_{h}\right)=\hat{L}_{h}\left(\hat{\varphi}_{h}\right), \quad \forall \hat{\varphi}_{h} \in \hat{Y}_{h} .
\end{array}\right.
$$

where $\hat{a}_{h}$ is a the bilinear form defined by

$$
\hat{a}_{h}\left(v_{h}, \hat{\varphi}_{h}\right)=\int_{\Omega} \nabla_{H} v_{h} \nabla_{H} \hat{\varphi}_{h}+\int_{\Omega} \partial_{z}\left(\hat{\mathcal{T}}_{h}\left(v_{h}\right)\right) \partial_{z} \hat{\varphi}_{h}
$$

with $\hat{\mathcal{T}}_{h}$ the mapping defined by $\hat{\mathcal{T}}_{h} v_{h}=\sum_{\alpha=1}^{N} v_{h}^{\alpha} \otimes \hat{\sigma}_{\alpha} \in \hat{Y}_{h}$, for $v_{h} \in X_{h}$, and $\hat{L}_{h}\left(\hat{\varphi}_{h}\right)=<f, \hat{\varphi}_{h}>_{\Omega}+\int_{\Gamma_{s}} g \hat{\varphi}_{h}-\hat{a}_{h}\left(g_{h}, \hat{\varphi}_{h}\right)$. We assume in particular $g \in H_{0}^{1}(\omega)$ in order to have this last term well defined. 
Observation 3. The basis functions of spaces $Y_{h}$ and $\hat{Y}_{h}$ only differ on the layers $\Omega_{N-1}$ and $\Omega_{N}$. Then the form $\hat{a}_{h}$ only differs from the form $a_{h}$ defined in (7) in the integrals on $\Omega_{N-1} \cup \Omega_{N}$. Then a slight modification of the proof of Lemma 1 yields

$$
\frac{4}{5}\left\|\nabla\left(\hat{\mathcal{T}}_{h} v_{h}\right)\right\|_{0, \Omega} \leq\|\| v_{h}\|\|_{X_{h}} \leq 4\left\|\nabla\left(\hat{\mathcal{T}}_{h} v_{h}\right)\right\|_{0, \Omega}, \forall v_{h} \in X_{h} .
$$

Consequently, the mapping $\hat{\mathcal{T}}_{h}$ is too an isomorphism between the normed spaces $X_{h}$ and $\hat{Y}_{h}$. This justifies the choice of the new norm on space $X_{h}$.

\subsection{Well-posedness and convergence analysis}

In this section we first study, analogously as we see in Section 3, the wellposedness of the multilayer problem 45):

Theorem 9. We assume that $g \in H_{0}^{1}(\omega)$, then the multilayer problem (45) admits a unique solution $v_{h} \in X_{h}$ that satisfies the estimate

$$
\left\|\left|v_{h}\right|\right\|_{X_{h}} \leq \frac{5}{2}\left(\|f\|_{H^{-1}(\Omega)}+(\sqrt{L}+2 \sqrt{h})\|g\|_{0, \omega}+h \sqrt{h}\left\|\nabla_{H} g\right\|_{0, \omega}\right) .
$$

Proof: (Sketch) Reasoning as in the proof of Theorem 1 for the multilayer problem (8), we deduce that the form $\hat{a}_{h}$ is stable and satisfies the inf-sup condition

$$
\inf _{v_{h} \in X_{h}} \sup _{\hat{\varphi}_{h} \in \hat{Y}_{h}} \frac{\hat{a}_{h}\left(v_{h}, \hat{\varphi}_{h}\right)}{\left\|\nabla \hat{\varphi}_{h}\right\|_{0, \Omega}\left|\left\|v_{h} \mid\right\|_{X_{h}}\right.} \geq \frac{2}{5} .
$$

This new inf-sup condition is proved similarly to (20), as a consequence of estimates (46) and taking into account the differences between the forms $a_{h}$ and $\hat{a}_{h}$ mentioned in Observation 3

Then problem (45) admits a unique solution that depends continuously on the data $f$ and $g$. The estimate (47) follows from (48), and standard estimates of $\hat{L}_{h}\left(\hat{\varphi}_{h}\right)$.

Finally we prove, analogously again to the case of the Poisson problem (2), the convergence of the discrete solution of the multilayer system 45 to the weak solution of the variational problem 42 , so as optimal order error estimates for smooth solutions. For that, let us define the operator $\hat{\Pi}_{h}$ defined by $\hat{\Pi}_{h} v=$ $\Pi_{h} v+g_{h}$, for $v \in H_{b l}^{1}(\Omega)$, with $\Pi_{h}$ defined by (24). Then we have: 
Theorem 10. Assume that the weak solution of (42) satisfies $v \in H^{l+1}(\Omega) \cap$ $H_{b l}^{1}(\Omega)$, with $l \geq 1$. Then, the solution $v_{h} \in X_{h}$ of the discretization 45. verifies

$$
\left.\left\|\hat{\Pi}_{h} v-v_{h}\right\|\right|_{X_{h}} \leq C\left(\left(h+k^{l}\right)\|v\|_{l+1, \Omega}+\sqrt{h}\|g\|_{0, \omega}+h \sqrt{h}\left\|\nabla_{H} g\right\|_{0, \omega}\right)
$$

for some constant $C>0$ a constant.

Moreover, if the solution $v$ of problem (42) is only in $H_{b l}^{1}(\Omega)$, then it holds

$$
\lim _{h \rightarrow 0}||\left|\hat{\Pi}_{h} v-v_{h}\right| \|_{X_{h}}=0 .
$$

\section{Numerical results}

In this section we present some numerical 3D experiments, to analyze the computing time reduction obtained by the parallel computation of each multilayer discretization, so as to test the error estimates. We have used the FreeFem++ software (cf. [17]).

Concretely, for the Poisson problem (1), we solve the multilayer problem (8) by an iterative procedure. Taking the test functions $\varphi_{h}=\varphi^{\alpha} \otimes \sigma_{\alpha}, 1 \leq \alpha \leq N$ in (8), this problem is equivalent to the following linear system, with unknowns $v_{h}^{\alpha}, \alpha=1, \cdots, N \in V_{0, k}(\omega)$ :

$$
\begin{aligned}
& \int_{\omega}\left(\frac{5 h}{8} \nabla_{H} v_{h}^{1}+\frac{h}{8} \nabla_{H} v_{h}^{2}\right) \cdot \nabla_{H} \varphi^{1}+\int_{\omega}\left(\frac{3}{h} v_{h}^{1}-\frac{1}{h} v_{h}^{2}\right) \varphi^{1}= \\
& =L\left(\varphi^{1} \otimes \sigma_{1}\right), \quad \forall \varphi^{1} \in V_{0, k}(\omega) ; \\
& \int_{\omega}\left(\frac{h}{8} \nabla_{H} v_{h}^{\alpha-1}+\frac{3 h}{4} \nabla_{H} v_{h}^{\alpha}+\frac{h}{8} \nabla_{H} v_{h}^{\alpha+1}\right) \cdot \nabla_{H} \varphi^{\alpha}+ \\
& +\int_{\omega}\left(-\frac{1}{h} v_{h}^{\alpha-1}+\frac{2}{h} v_{h}^{\alpha}-\frac{1}{h} v_{h}^{\alpha+1}\right) \varphi^{\alpha}=L\left(\varphi^{\alpha} \otimes \sigma_{\alpha}\right), \quad \forall \varphi^{\alpha} \in V_{0, k}(\omega), \\
& \alpha=2, \cdots, N-1 ; \\
& \int_{\omega}\left(\frac{h}{8} \nabla_{H} v_{h}^{N-1}+\frac{5 h}{8} \nabla_{H} v_{h}^{N}\right) \cdot \nabla_{H} \varphi^{N}+\int_{\omega}\left(-\frac{1}{h} v_{h}^{N-1}+\frac{3}{h} v_{h}^{N}\right) \varphi^{N}= \\
& =L\left(\varphi^{N} \otimes \sigma_{N}\right), \quad \forall \varphi^{N} \in V_{0, k}(\omega) .
\end{aligned}
$$


We solve this linear system through a block-Jacobi iterative algorithm by layers, that leads to solve at each iteration the following sequence of $2 \mathrm{D}$ horizontal problems :

$$
\begin{aligned}
& \int_{\omega} \frac{5 h}{8} \nabla_{H} v_{h}^{1, k+1} \cdot \nabla_{H} \varphi^{1}+\frac{3}{h} \int_{\omega} v_{h}^{1, k+1} \varphi^{1}=-\frac{h}{8} \int_{\omega} \nabla_{H} v_{h}^{2, k} \cdot \nabla_{H} \varphi^{1}+ \\
& +\frac{1}{h} \int_{\omega} v_{h}^{2, k} \varphi^{1}+\frac{5 h}{8} \int_{\omega} f^{1} \varphi^{1}+\frac{h}{8} \int_{\omega} f^{2} \varphi^{1}, \quad \forall \varphi^{1} \in V_{0, k}(\omega) ; \\
& \frac{3 h}{4} \int_{\omega} \nabla_{H} v_{h}^{\alpha, k+1} \cdot \nabla_{H} \varphi^{\alpha}+\frac{2}{h} \int_{\omega} v_{h}^{\alpha, k+1} \varphi^{\alpha}= \\
& -\frac{h}{8} \int_{\omega}\left(\nabla_{H} v_{h}^{\alpha-1, k}+\nabla_{H} v_{h}^{\alpha+1, k}\right) \cdot \nabla_{H} \varphi^{\alpha}+\frac{1}{h} \int_{\omega}\left(v_{h}^{\alpha-1, k}+v_{h}^{\alpha+1, k}\right) \varphi^{\alpha} \\
& +\int_{\omega}\left(\frac{h}{8} f^{\alpha-1}+\frac{3 h}{4} f^{\alpha}+\frac{h}{8} f^{\alpha+1}\right) \varphi^{\alpha}, \quad \forall \varphi^{\alpha} \in V_{0, k}(\omega) 2 \leq \alpha \leq N-1 ; \\
& \int_{\omega} \frac{5 h}{8} \nabla_{H} v_{h}^{N, k+1} \cdot \nabla_{H} \varphi^{N}+\frac{3}{h} \int_{\omega} v_{h}^{N, k+1} \varphi^{N}=-\frac{h}{8} \int_{\omega} \nabla_{H} v_{h}^{N-1, k} \cdot \nabla_{H} \varphi^{N}+ \\
& +\frac{1}{h} \int_{\omega} v_{h}^{N-1, k} \varphi^{1}+\frac{5 h}{8} \int_{\omega} f^{N} \varphi^{N}+\frac{h}{8} \int_{\omega} f^{N-1} \varphi^{N}, \quad \forall \varphi^{N} \in V_{0, k}(\omega),
\end{aligned}
$$

with $f^{\alpha}=\int_{\Omega} f \sigma_{\alpha}, \alpha=1, \cdots, N$.

This block-Jacobi algorithm is solved using an affine parallel GMRES algorithm (built-in in FreeFem ++ ), where each layer-wise 2D problem is solved in a different processor using basically the following function:

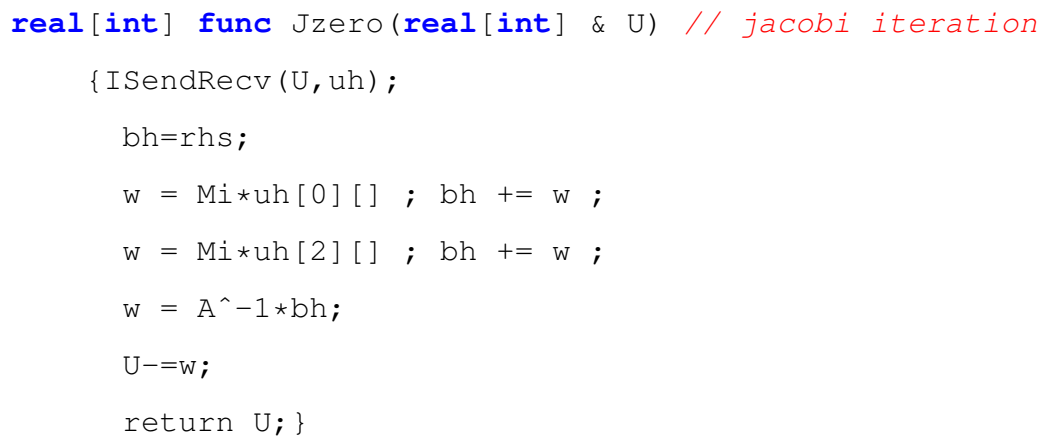

where, for example, to solve the 2D problem on layer $\alpha$ with $\alpha=2, \cdots, N-1$, rhs is the contribution of the integrals in $f$ in (51), Mi*uh[0] [] are the 
integrals in $v_{h}^{\alpha-1}$ in (51) and Mi*uh [2] [ ] are the integrals in $v_{h}^{\alpha+1}$.

The numerical tests have been run on Altix UV 2000 with 32 CPUs Intel Xeon 64 bits EvyBridge E4650 with 10 core with a total of 2048 Gb RAM, using the same number of processors as of layers.

\section{Test 1: Homogeneous Dirichlet boundary conditions}

In this numerical test, we consider a smooth exact solution

$$
v(x, y, z)=4 z^{2}(1-z) \sin \left(\pi x^{2}\right) \sin (\pi y)
$$

of 11 in $\Omega=] 0,1\left[{ }^{3}\right.$ with homogeneous Dirichlet boundary conditions. We compare the solution obtained by the block-Jacobi algorithm [51, with a piecewise affine finite element space $V_{0, k}(\omega)$ constructed on a structured horizontal grid of $\omega=] 0,1[2$, versus the direct solution of the global 3D problem (1) by the standard Galerkin method with piecewise affine finite elements on a structured tetrahedral grid, with the same degrees of freedom as $X_{h}$ (that is, number of layers $\left.\times \operatorname{dim}\left(X_{h}\right)\right)$.

Concretely, we at first compare the CPU time to obtain the sequential solution by a Conjugate Gradient Solver (built-in in FreeFem++ too) versus the mean CPU time required by the processors to solve the block-Jacobi algorithm (51).

For that, we compute two tests. Assume that the horizontal grid size is $k=1 / N H$ for some integer number $N H \geq 1$. In the first test we set $N Z=N H$ and progressively increase these numbers from $N Z=N H=10$ to $N Z=N H=$ 100, with step 10. In the second one, we fix the horizontal grid size to the value $N H=1 / 40$ and progressively increase the number of layers from $N Z=10$ to $N Z=100$, with step 10 .

Figure 4 shows for each of these two tests two ratios, on the one hand, the ratio between the $\mathrm{CPU}$ time to solve the sequential $3 \mathrm{D}$ problem by the Conjugate Gradient Solver (CG) versus the mean CPU time used by processors to build the matrix and the second member of the $2 \mathrm{D}$ system that we solve in each layer/processor and solve it by the affine parallel GMRES algorithm 


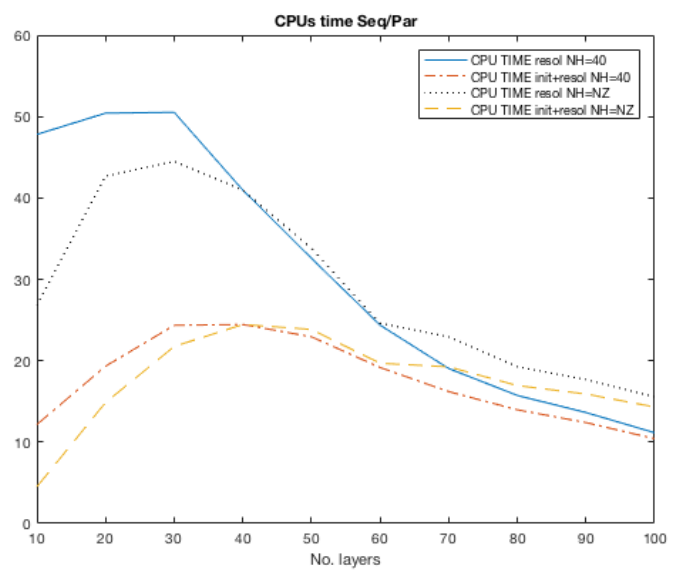

Figure 4: Test 1: Ratio sequential versus parallel CPU times.

(lines with legend "CPU TIME init+resol"). On the other hand, the ratio between the CPU time to solve the sequential problem by the CG Solver versus the mean CPU time used by processors only to solve the $2 \mathrm{D}$ system in each layer/processor by the affine parallel GMRES algorithm (lines with legend "CPU TIME resol")

We notice that we obtain similar results for both tests. As the number of layers increases, there is a large gain of computing time, up to an optimal rate around 30/40 layers, that deteriorates as $N Z$ increases from this value.

Furthermore, we test the error estimates. For that, Table 1 shows the obtained results for the relative errors in the $L^{2}(\Omega)$ and the $H^{1}(\Omega)$ discrete norms introduced in 10 , for $N Z=10,20,40,80$ and $k=1 / N Z$, thus with the same grid size in the horizontal and vertical directions. Also, Table 1 displays the estimated orders of convergence, computed from these errors and the number of iterations used by the affine parallel GMRES algorithm to solve the 2D system in each layer/processor.

In all test we denote by $\left\|v_{h}\right\|_{1, h}=\left\|v_{h}\right\|_{X_{h}}$ and $\left\|v_{h}\right\|_{0, h}=\left(h \sum_{\alpha=1}^{N}\left\|v_{h}^{\alpha}\right\|_{0, \omega}^{2}\right)^{1 / 2}$, and we compute $\operatorname{ord}_{k, h}=\log _{2}\left(\frac{\left\|e_{h}\right\|_{k, h}}{\left\|e_{h / 2}\right\|_{k, h / 2}}\right)$ for $k=0,1$. 


\begin{tabular}{|c|c|c|c|c|}
\hline $\mathrm{N}$ & 10 & 20 & 40 & 80 \\
\hline$\left\|e_{h}\right\|_{0, h}$ & 0.0311414 & 0.0077288 & 0.00192875 & 0.000481821 \\
\hline$\left\|e_{h}\right\|_{1, h}$ & 0.162031 & 0.080199 & 0.0399963 & 0.0199851 \\
\hline GMRES iters & 21 & 39 & 89 & 252 \\
\hline ord $_{0, h}$ & 2.0105 & 2.0026 & 2.0011 & - \\
\hline ord $_{1, h}$ & 1.0146 & 1.0037 & 1.009 & - \\
\hline
\end{tabular}

Table 1: Test 1 , relative errors in discrete $L^{2}(\Omega)$ and $H^{1}(\Omega)$ norms and estimated convergence orders.

We obtain first order convergence in the $H^{1}(\Omega)$ discrete norm, in full agreement with our theoretical expectations. We also obtain second order convergence in the $L^{2}(\Omega)$ discrete norm, as arises for Galerkin finite element solutions of regular elliptic problems.

We obtain quite similar convergence orders if we use unstructured horizontal meshes, that we not display for brevity.

\section{Test 2: Domains with non-flat surface}

Now we present a numerical test, where we consider a computational domain $\hat{\Omega}$ that is obtained from the reference domain $\Omega=] 0,1\left[{ }^{3}\right.$ by the change of variables 32 with the function $\eta$ given by

$$
\eta(x, y)=1+\epsilon \sin (2 \pi(x+y)) .
$$

This function satisfies 33 if $\epsilon<\frac{1}{2 \sqrt{2} \pi}$.

In this test we compare an exact solution of the Laplace problem 34 in $\hat{\Omega}$ with the solution obtained by our multilayer discretization 36 in the unit cube $\Omega$. Concretely, we consider the function

$$
u(x, y, z)=64 \sin \left(\frac{\pi z}{\eta(x, y)}\right) x^{2}(1-x) y(1-y)^{2}
$$

that is the exact solution for the Laplace problem 34 in $\hat{\Omega}$, and we consider the previous function $\eta$ with $\epsilon=0.15$. Figure 5 shows the global 3D Galerkin solution of (34) with $h=1 / 36$. 


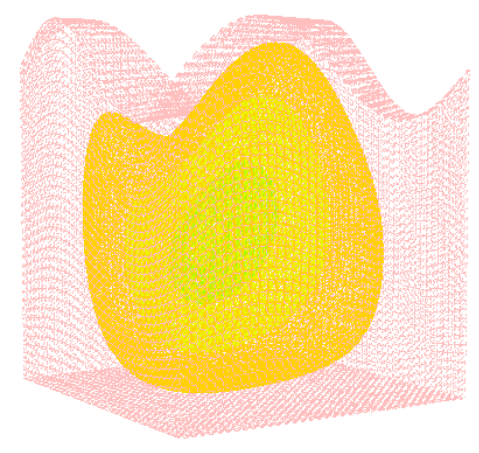

Figure 5: Test 2. Solution of problem 34 with 36 layers.

Figure 6 shows the same two CPU ratios for each of the two tests shown in Figure 4

We observe a similar behavior as in Test 1 , there is an increasing computational gain as the number of processors increases up to 40/50 layers (lines with legend "CPU TIME init+resol"), that further progressively deteriorates. The gain is somewhat higher than in the previous test, possibly due to the larger computational cost to obtain the 3D sequential global solution in the computational domain $\hat{\Omega}$.

Also, Table 2 display the norms of the relative errors, estimated orders of convergence, as well as the number of iterations used by GMRES, for $N Z=$ $10,20,40,80$. We observe a quite similar behavior as in Test 1 , we recover the theoretical first order in the $H^{1}(\Omega)$ discrete norm, and second order in the $L^{2}(\Omega)$ discrete norm. Here we do the tests for structured horizontal meshes, but we again obtain quite similar results if we use unstructured horizontal meshes.

\section{Test 3: Dirichlet-Neumann boundary conditions}

In this third test, we consider an exact solution of (41) in $\Omega$ with Neumann boundary conditions on $\Gamma_{s}$ and homogeneous Dirichlet boundary conditions on 


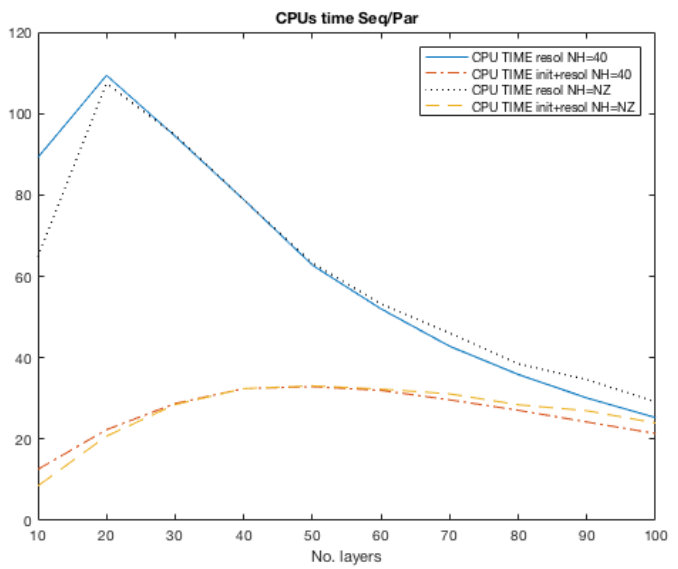

Figure 6: Test 2: Ratio sequential versus parallel CPU times.

\begin{tabular}{|c|c|c|c|c|}
\hline $\mathrm{N}$ & 10 & 20 & 40 & 80 \\
\hline$\left\|e_{h}\right\|_{0, h}$ & 0.0284935 & 0.00677639 & 0.00158417 & 0.000390097 \\
\hline$\left\|e_{h}\right\|_{1, h}$ & 0.162359 & 0.0786338 & 0.0381604 & 0.0189835 \\
\hline GMRES iters & 24 & 46 & 117 & 355 \\
\hline ord $_{0, h}$ & 2.0720 & 2.0968 & 2.0218 & - \\
\hline ord $_{1, h}$ & 1.0460 & 1.0431 & 1.0073 & - \\
\hline
\end{tabular}

Table 2: Test 2, relative errors in discrete $L^{2}(\Omega)$ and $H^{1}(\Omega)$ norms and estimated convergence orders.

$\Gamma_{b} \cup \Gamma_{l}:$

$$
v(x, y, z)=64 \sin \left(\frac{\pi z}{2}\right) x^{2}(1-x) y(1-y)^{2} .
$$

Figure 7 shows the global 3D Galerkin solution of 41) with $h=1 / 36$.

Figure 8 shows shows the same two CPU ratios for each of the two tests as in Figure 4 and Figure 6

We observe a similar behavior, there is an increasing computational gain as the number of processors increases to around 30/40 layers, that further progressively deteriorates. The gain is somewhat smaller as for the Test 1 due to 
$\mathrm{NZ}=36$, uhmax $=1.40308$

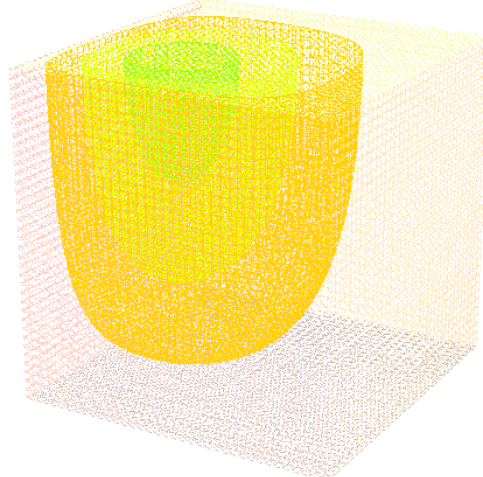

Figure 7: Test 3. Solution of problem 41 with 36 layers.

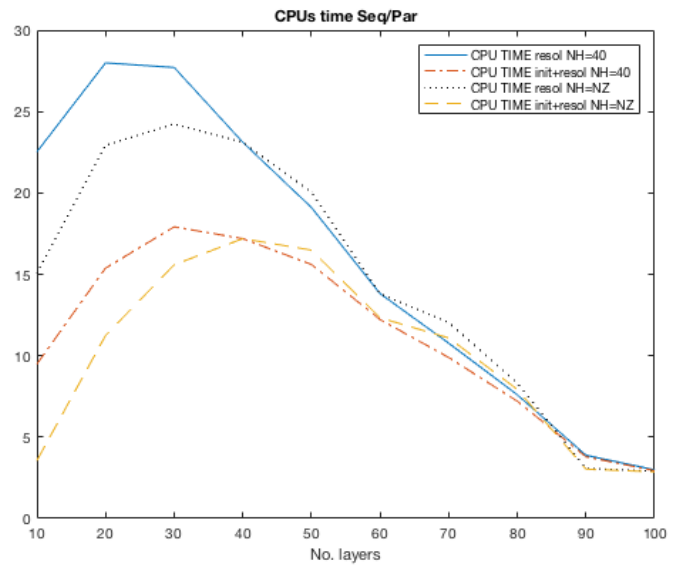

Figure 8: Test 3. Ratio multilayer versus Galerkin 3D CPU times. 
additional calculations required in the upper layers.

Also, Table 3 displays the norms of the relative errors, estimated orders of convergence and number of GMRES iterations, for structured horizontal meshes, again for $N Z=10,20,40,80$. We observe a quite similar behavior to the Test 1 that qualitatively are the same for unstructured horizontal meshes.

\begin{tabular}{|c|c|c|c|c|}
\hline $\mathrm{N}$ & 10 & 20 & 40 & 80 \\
\hline$\left\|e_{h}\right\|_{0, h}$ & 0.0423782 & 0.0104868 & 0.00261555 & 0.000654319 \\
\hline$\left\|e_{h}\right\|_{1, h}$ & 0.21214 & 0.10533 & 0.0526301 & 0.0263241 \\
\hline GMRES iters & 22 & 44 & 122 & 635 \\
\hline ord $_{0, h}$ & 2.0147 & 2.0034 & 1.9990 & - \\
\hline ord $_{1, h}$ & 1.0101 & 1.0010 & 0.9995 & - \\
\hline
\end{tabular}

Table 3: Test 3, relative errors in discrete $L^{2}(\Omega)$ and $H^{1}(\Omega)$ norms and estimated convergence orders.

\section{Conclusions}

We have studied in this paper a multilayer discretization of second order elliptic problems, aimed at providing reliable multilayer discretizations of shallow fluid flow problems with diffusive effects. This is a Petrov-Galerkin discretization in which the trial functions are piecewise constant per horizontal layers, while the trial functions are continuous piecewise linear, on a vertically shifted grid.

We have introduced the discretization for the Poisson problem with Dirichlet boundary conditions in cylindric domains, and extended it to Neumann boundary conditions in cylindric domains, so as to domains with variable surface, that appear in free-surface fluid flow problems. We have proved the well posedness and optimal error order estimates for these three discretizations in natural norms, based upon specific inf-sup conditions.

We have performed numerical tests with parallel computing of the solution by a block-Jacobi algorithm, based upon the multilayer structure of the 
discretization, for academic problems with smooth solutions. We recover the theoretical optimal error order convergence, and observe a high increase of the speed of computations for a moderate number of processors. This confirms the interest of applying the technique introduced to multilayer discretizations of fluid flow problems.

In further steps we shall improve the parallelization procedure to obtain a good scaling of the CPU computing time for large number of processors. Also, we will apply the discretization introduced to convection-diffusion problems, and then to multilayer discretizations of Navier-Stokes and related equations. We will also extend the discretization to higher-order approximations of the unknowns. These works are now in progress.

\section{Acknowledgements}

This research was partially supported by "Proyecto de Excelencia de la Junta de Andalucía” under grant P12-FQM-454.

\section{References}

\section{References}

[1] L. Bonaventura, E. D. Fernández-Nieto, J. Garres-Díaz, G. Narbona-Reina, Multilayer shallow water models with locally variable number of layers and semi-implicit time discretization, Journal of Computational Physics 364 (2018) $209-234$.

URL https://www.sciencedirect.com/science/article/pii/ S0021999118301694

[2] E. D. Fernández-Nieto, J. Garres-Díaz, A. Mangeney, G. Narbona-Reina, A multilayer shallow model for dry granular flows with the $\mu(I)$ rheology: Application to granular collapse on erodible beds, Journal of Fluid Mechanics 798 (2016) 643-681. 
[3] J. Sainte-Marie, Vertically averaged models for the free surface nonhydrostatic euler system: derivation and kinetic interpretation, Math. Models Methods Appl. Sci. 21 (3) (2011) 459-490.

[4] B. Cockburn, S. Chi-Wang, The local discontinuous galerkin method for time-dependent convection-diffusion systems, SIAM J. Numer. Anal. 35 (6) (1998) 2440-2463.

[5] D. N. Arnold, F. Brezzi, B. Cockburn, L. D. Marini, Unified analysis of discontinuous galerkin methods for elliptic problems, SIAM J. Numer. Anal. 39 (5) (2002) 1749-1779.

[6] D. A. Di Pietro, A. Ern, Mathematical Aspects of Discontinuous Galerkin Methods, Vol. 69 of Mathématiques \& Applications, Springer-Verlag, Berlin, 2011.

[7] C. L. Bottasso, S. Micheletti, R. Sacco, The discontinuous petrov-galerkin method for elliptic problems, Comput. Methods Appl. Mech. Engrg. 191 (31) (2002) 3391-3409.

[8] P. Causin, R. Sacco, A discontinuous petrov-galerkin method with lagrangian multipliers for second order elliptic problems, SIAM J. Numer. Anal. 43 (1) (2005) 280-302.

[9] L. Demkowicz, J. Gopalakrishnan, A class of discontinuous petrov-galerkin methods. part i: The transport equation, Comput. Methods Appl. Mech. Engrg. 199 (23-24) (2010) 1558-1572.

[10] L. Demkowicz, J. Gopalakrishnan, Analysis of the dpg method for the poisson problem, SIAM J. Numer. Anal. 49 (5) (2011) 1788-1809.

[11] L. Demkowicz, J. Gopalakrishnan, A class of discontinuous petrov-galerkin methods. part ii: Optimal test functions, Numer. Meth. Part. Diff. Equa. 27 (1) (2011) 70-105. 
[12] L. Demkowicz, J. Gopalakrishnan, Discontinuous petrov-galerkin (dpg) method, ICES Report 15-20 (2015) 1-21.

[13] H. Brezis, Functional Analysis, Sobolev Spaces and Partial Differential Equations, Springer-Verlag, New York, 2011.

[14] A. Ern, J.-L. Guermond, Theory and practice of finite element methods, Vol. 159 of Applied Mathematical Sciences, Springer-Verlag, New York, 2004.

[15] C. Bernardi, Y. Maday, F. Rapetti, Discrétisations variationnelles de problèmes aux limites elliptiques, Vol. 45 of Mathématiques \& Applications, Springer-Verlag, Berlin, 2004.

[16] P. Clément, Approximation by finite element functions using local regularization, Rev. Française Automat. Informat. Recherche Opérationnelle Sér. Rouge Anal. Numér. 9 (R-2) (1975) 77-84.

[17] F. Hecht, S. Auliac, O. Pironneau, J. Morice, A. Le Hyaric, K. Ohtsuka, P. Jolivet, Freefem++, third edition, version 3.58-1 (2018).

URL http://www. freefem.org/fft+/ftp/freefem++doc.pdf 